\title{
Extensional rheology and elastic instabilities of a wormlike micellar solution in a microfluidic cross-slot device $\dagger+$
}

\author{
Simon J. Haward, ${ }^{* a}$ Thomas J. Ober, ${ }^{a}$ Mónica S.N. Oliveira, ${ }^{b}$ Manuel A. Alves ${ }^{b}$ and Gareth H. McKinley ${ }^{a}$ \\ Received 3rd August 2011, Accepted 16th September 2011 \\ DOI: 10.1039/c1sm06494k
}

\begin{abstract}
Wormlike micellar surfactant solutions are encountered in a wide variety of important applications, including enhanced oil recovery and ink-jet printing, in which the fluids are subjected to high extensional strain rates. In this contribution we present an experimental investigation of the flow of a model wormlike micellar solution (cetyl pyridinium chloride and sodium salicylate in deionised water) in a well-defined stagnation point extensional flow field generated within a microfluidic cross-slot device. We use micro-particle image velocimetry ( $\mu$-PIV) and full-field birefringence microscopy coupled with macroscopic measurements of the bulk pressure drop to make a quantitative characterization of the fluid's rheological response over a wide range of deformation rates. The flow field in the micromachined cross-slot is first characterized for viscous flow of a Newtonian fluid, and $\mu$-PIV measurements show the flow field remains symmetric and stable up to moderately high Reynolds number, $R e \approx 20$, and nominal strain rate, $\dot{\varepsilon}_{n o m} \approx 635 \mathrm{~s}^{-1}$. By contrast, in the viscoelastic micellar solution the flow field remains symmetric only for low values of the strain rate such that $\dot{\varepsilon}_{\text {nom }} \leq \lambda_{M}{ }^{-1}$, where $\lambda_{M}=2.5 \mathrm{~s}$ is the Maxwell relaxation time of the fluid. In this stable flow regime the fluid displays a localized and elongated birefringent strand extending along the outflow streamline from the stagnation point, and estimates of the apparent extensional viscosity can be obtained using the stressoptical rule and from the total pressure drop measured across the cross-slot channel. For moderate deformation rates $\left(\dot{\varepsilon}_{n o m} \geq \lambda_{M}{ }^{-1}\right)$ the flow remains steady, but becomes increasingly asymmetric with increasing flow rate, eventually achieving a steady state of complete anti-symmetry characterized by a dividing streamline and birefringent strand connecting diagonally opposite corners of the cross-slot. Eventually, as the nominal imposed deformation rate is increased further, the asymmetric divided flow becomes time dependent. These purely elastic instabilities are reminiscent of those observed in crossslot flows of polymer solutions, but seem to be strongly influenced by the effects of shear localization of the micellar fluid within the microchannels and around the re-entrant corners of the cross-slot.
\end{abstract}

\section{Introduction}

Wormlike micelles are elongated semi-flexible aggregates resulting from the self-assembly of amphiphilic molecules in solution. ${ }^{1,2}$ In the semi-dilute and concentrated regimes, wormlike micellar solutions are entangled and exhibit viscoelasticity in a way similar to polymer solutions, with a typical relaxation time ( $\lambda$ ) on the order of milliseconds to seconds. ${ }^{3-6}$ However,

${ }^{a}$ Hatsopoulos Microfluids Laboratory, Department of Mechanical Engineering, Massachusetts Institute of Technology, 77 Massachusetts Avenue, Cambridge, MA, 02139, USA.E-mail: shaward@MIT.EDU; Tel: +16172530273

${ }^{b}$ Departamento de Engenharia Química, CEFT, Faculdade de Engenharia da Universidade do Porto, Rua Dr Roberto Frias s/n, 4200-465 Porto, Portugal

$\dagger$ Electronic supplementary information (ESI) available: See DOI: $10.1039 / \mathrm{c} 1 \mathrm{sm} 06494 \mathrm{k}$

† Dedication: This article is dedicated to Dr Jeff Odell: may he enjoy a long and very happy retirement. a significant difference between wormlike micelles and conventional polymers with covalently bonded back-bone chains is their ability to break and reform dynamically, allowing stress relaxation to occur through mechanisms additional to reptation, ${ }^{3-6}$ and allowing the rheological properties of the solutions to recover subsequent to degradation in strong flows. The resulting unique properties of such fluids have led to their use in a wide range of applications in household and industrial fluids. ${ }^{7,8}$ Wormlike micellar solutions are becoming increasingly important in jetting and spraying applications, ${ }^{9-11}$ turbulent drag reduction, ${ }^{12,13}$ enhanced oil recovery ${ }^{14-16}$ and the formulation of many consumer products (e.g. paints, cosmetics, detergents) in which particle suspension/sedimentation and/or rise of air bubbles is a concern. ${ }^{17,18}$ Such processes involve complex flow fields with strongly extensional components, which can cause large and rapid deformation of the fluid microstructure. The resulting stretching and alignment of micelles leads to a range of poorly understood phenomena including flow-induced chain scission 
and structure formation, ${ }^{19-22}$ and elastic instabilities. ${ }^{23,24} \mathrm{~A}$ comprehensive recent review of strong extensional flows of wormlike micellar solutions, with a particular emphasis on industrial applications and viscoelastic flow instabilities, has been provided by Rothstein. ${ }^{25}$

Extensional flows of wormlike micellar solutions involve the convergence of two fundamental concepts underlying our understanding of viscoelastic flows: flow-induced microstructural modifications $^{26}$ and purely elastic flow instabilities. ${ }^{27,28}$ Recent studies on flows of polymer solutions have largely focussed on elastic instabilities, ${ }^{27,28}$ while studies on wormlike micelles have concentrated on the phenomenon of shear banding. ${ }^{26,29}$ For a comprehensive discussion of these phenomena we refer to a number of excellent recent reviews on the subjects. ${ }^{26,28,30}$ Here we focus on reviewing the background to extensional flows of wormlike micellar solutions and we present our motivations for investigating elastic instabilities in such flows.

Early measurements of the uniaxial extensional rheology of a variety of micelle forming surfactant/counterion systems in the commercial Rheometrics RFX opposed jets device showed an increase in the extensional viscosity of the fluids as the flow rate was increased beyond a critical value. ${ }^{31-34}$ The critical flow rate broadly corresponded to an extensional strain rate, $\dot{\varepsilon}$, on the order of $1 / \lambda$, where $\lambda$ is the fluid relaxation time, such that the critical Weissenberg number, $W i=\dot{\varepsilon} \lambda \sim 1$. In addition, flowinduced birefringence observations, ${ }^{32}$ and especially light scattering measurements, ${ }^{34}$ on the flowing fluids showed a clear correspondence between the extensional viscosity and the degree of micellar orientation and radius of gyration. These observations were consistent with a micellar coil-stretch transition, as originally predicted by De Gennes ${ }^{35}$ for high molecular weight flexible polymers, which has been demonstrated convincingly in extensional flow studies with dilute solutions of flexible polymers. ${ }^{36-39}$ Moreover, a reduction in the extensional viscosity of the wormlike micellar solutions above a second, higher critical Weissenberg number, corresponded to a reduction in the radius of gyration of micelles, possibly indicating their flowinduced fracture. ${ }^{34}$ This same second critical $W i$ also marked the onset of a flow instability ${ }^{32}$ similar to the birefringent 'flare' observed in semidilute polymer solutions, ${ }^{38}$ and likely led to the disruption of the well-defined stagnation point between the jets. ${ }^{33}$

Recent work by Stone et al. $^{40}$ used flow in a cross-slot geometry, coupled with fluorescence microscopy, to directly visualize the dynamics of individual wormlike micelles formed from a diblock copolymer of poly(ethylene oxide) and polybutadiene. Cross-slot geometries consist of orthogonal bisecting channels with opposing inlets and outlets, such that a planar extensional flow is generated along the in-flowing and out-flowing symmetry plane, with a stagnation point at the centre of the cross. ${ }^{41}$ The results with wormlike micelles were highly reminiscent of similar experiments performed using fluorescently labelled DNA. ${ }^{36,39}$ Micelles were shown to stretch above flow rates corresponding to a critical $W i \sim 1$, reaching highly extended configurations after sufficient time in the extensional flow field. Higher values of $W i$ resulted in more affine deformation of micelles with the surrounding fluid, although such conditions also resulted in an increased occurrence of micelles becoming trapped in metastable kinked and folded conformations.
One of the motivations for our present work stems from the recent study by Arratia et al., ${ }^{42}$ using microfluidic cross-slots with dilute polymer solutions to demonstrate a new type of purely elastic flow instability. Such microscale devices only require small volumes of test fluid and low volume flow rates but can achieve significant extensional strain rates and Weissenberg numbers. The combination of small length scale and correspondingly low characteristic velocities results in a vanishing Reynolds number $(R e)$ meaning that inertial effects can usually be neglected in such devices. ${ }^{43}$ Arratia et al. ${ }^{42}$ used an elastic solution of a flexible high molecular weight polyacrylamide, to show that beyond a critical Weissenberg number, $W i \approx 4.5$, the flow field in the cross-slots became spatially asymmetric while remaining steady in time. The asymmetry intensified as a function of $W i$, indicating a supercritical pitchfork bifurcation. ${ }^{27}$ At higher $W i>12.5$ the asymmetric flow became unsteady and time-dependent. All the experiments were conducted at $R e<10^{-2}$. The main features of the experiment were captured in subsequent simulations by Poole et al. ${ }^{44}$ using the upper-convected Maxwell (UCM) model, and more recently by Rocha $e t$ al. with a finitely extensible nonlinear elastic (FENE) dumbbell model. ${ }^{45}$ However, the instability mechanism is still a matter of debate. While Poole et al. advocated a macroscopic mechanism based on the compressive incoming kinematics, ${ }^{44} \mathrm{Xi}$ et al. proposed an essentially microscopic mechanism, where the instability is dominated by the physics at the stagnation point. ${ }^{46}$ Elastic instabilities in polymer solutions have demonstrable practical applications. They have been utilized in the design of microfluidic analogues of electronic circuit elements, such as resistors and bistable flip-flops,${ }^{47}$ and diodes or valves, ${ }^{48}$ and also have potential for efficient, low $R e$ mixing applications. ${ }^{42,49}$

The recent work of Pathak and Hudson ${ }^{23}$ used a cross-slot geometry to perform a detailed quantitative study of the flowinduced birefringence in two entangled wormlike micellar solutions. One was an aqueous solution of $100 \mathrm{mM}$ cetylpyridinium chloride $(\mathrm{CPyCl}) / 60 \mathrm{mM}$ sodium salicylate $(\mathrm{NaSal})$ and the other an aqueous solution of $30 \mathrm{mM}$ cetyltrimethylammonium bromide (CTAB)/240 mM NaSal. In both solutions intense and sharply-peaked bands of birefringence were observed around the stagnation point for $W i>0.5$. In the $\mathrm{CTAB} / \mathrm{NaSal}$ solution the birefringence decayed exponentially along the outflow axis of the cross-slot with a characteristic decay time similar to the fluid relaxation time. At higher $W i>1$ a flow bifurcation was observed similar to that reported by Arratia et al. for a semi-dilute polymer solution. ${ }^{42}$ Also consistent with Arratia et al., the bifurcation became unstable and time dependent at much higher $W i$. However, while the bifurcation in the CTAB solution showed the characteristic square-root dependence on $W i$ near the critical value (as also shown by polymer solutions ${ }^{42,44}$ ), the bifurcation in the $\mathrm{CPyCl}$ solution showed a different, and unexplained, scaling.

In the present study we focus our attention on the cross-slot flow of a $100 \mathrm{mM} \mathrm{CPyCl} / 60 \mathrm{mM}$ NaSal solution similar to that previously studied by Pathak and Hudson, ${ }^{23}$ but we present more detailed experimental results obtained over a much wider range of $W i$. We also use a cross-slot of much smaller characteristic dimension, to definitively obviate inertial effects. Our cross-slot has a deep cross-section to generate approximately 2D flow, so that extension-induced birefringence at the stagnation point dominates that induced by shear along the walls. We present detailed 
velocimetry measurements made over the same range of $W i$ as our birefringence measurements, and we couple these full-field data with bulk pressure drop measurements across the cross-slots to perform extensional viscometry. In Section 2 we provide details of the test cell geometry, its fabrication and our experimental methods. We also characterize the test fluid using cone-and-plate viscometry, microfluidic shear rheometry and capillary break-up extensional rheometry (CaBER). In Section 3, to fully characterize the kinematics generated by our microfluidic cross-slot design, we provide a detailed description of Newtonian flow in the test cell and compare the experimental results with numerical expectations. We then proceed to present detailed experimental results obtained with the wormlike micellar test fluid, which we discuss in the context of purely elastic instabilities.

\section{Experimental}

\subsection{Cross-slot fabrication and geometry}

An optical micrograph of the cross-slot geometry used in the study is shown in Fig. 1a. We define the $x$ and $y$ axes as shown in the figure, with the stagnation point (marked by the blue ' $x$ ' at the centre of the cross) taken as the origin of coordinates and the $z$-axis normal to the plane of the page. The slots have a width of $w=0.2 \mathrm{~mm}$, and a depth in the $z$-direction of $d=1$ $\mathrm{mm}$, providing an aspect ratio $\alpha=d / w=5$ and hence a quasi-2D flow. The length of the channels is $l=1.2 \mathrm{~mm}$. In all the results presented herein the inflow and outflow directions are as marked in Fig.1a.

The cross-slots were cut through a $2 \mathrm{~cm}$ diameter disc of 316 stainless steel using wire electrical discharge machining (wireEDM), which results in highly parallel, non-tapering walls and a smooth surface finish; features which are essential for producing a stable, symmetric flow. To create enclosed rectangular flow channels allowing optical access to the region near the stagnation point, $2 \mathrm{~mm}$ thick soda glass windows were glued to the front and rear surfaces of the stainless steel disc (see Fig. 1b). The glass windows were annealed overnight at $300{ }^{\circ} \mathrm{C}$ to remove residual optical anisotropies that could interfere with flowinduced birefringence measurements. The rear window had four holes drilled through ultrasonically, to allow the flow of fluid into/out of the cross-slot. Finally, the stainless steel/glass sandwich assembly was glued onto a monolithic stainless steel backplate, which made connections to the external plumbing and flow system, Fig. 1c. All bonds were made using silicone aquarium adhesive. Following the assembly of the cross-slots, the total depth of the flow cell (i.e. the gap between the inside surfaces of the glass windows) was measured using a confocal laser micrometre, providing an accurate value of $d=1050 \pm 10 \mu \mathrm{m}$. Thus, the thickness of the adhesive layer on either surface was $\approx 25 \pm 5 \mu \mathrm{m}$.

The same cross-slots have been used in a number of previous studies with a range of complex fluids in which an oscillatory extensional flow system was utilized. ${ }^{50-53}$ However, in the present study we use the continuous flow-through system illustrated in Fig. 2. The reasons for this are: (a) entangled wormlike micellar fluids can take considerable time to settle to 'steady-state" flow conditions, which can only be achieved in the oscillatory flow system for very low imposed flow rates, and (b) here we are interested in studying (possibly time-dependent) elastic
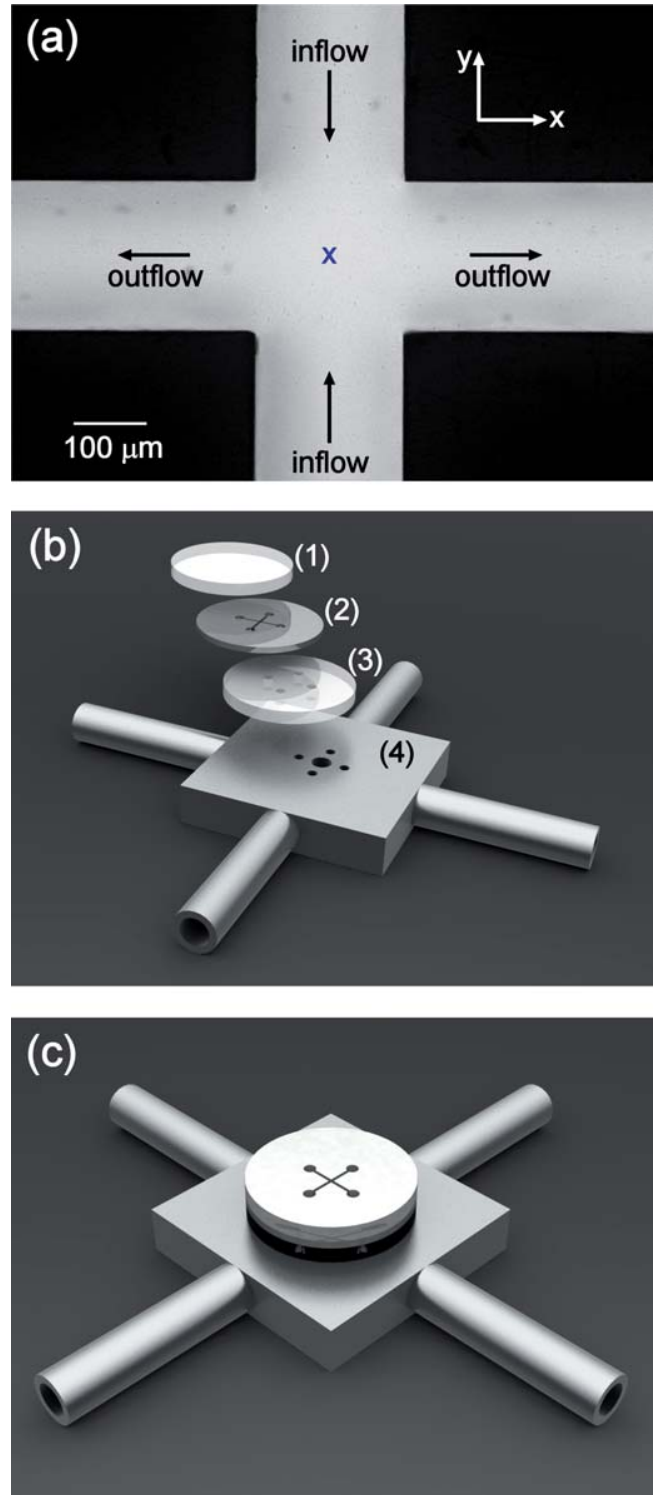

Fig. 1 (a) Micrograph of cross-slot, cut from $1 \mathrm{~mm}$ thick stainless steel by wire electrical discharge machining (wire-EDM). (b) Exploded schematic illustration of cross-slot flow-cell showing (1) glass front window, (2) stainless-steel cross-slot, (3) glass rear window with holes to permit fluid flow, (4) stainless steel flow-cell mount with four pipe connections to the flow system and a central "thru" hole to allow optical access to the stagnation point. (c) Illustration of cross-slot flow-cell, assembled using silicone adhesive.

instabilities with a time scale that is unknown a priori and this would be complicated if we imposed an oscillating flow field.

The continuous flow was generated using a Harvard PHDUltra syringe pump. The flow from the syringe (1) was split into two channels to provide the opposing inlet flow to the cross-slot (3). The two outlets were connected together so that they would be at the same pressure as each other and thus not lead to an unbalanced flow. One of the inlet channels was fitted with a GE Druck 5 psi gauge pressure transmitter (2) to measure the pressure drop across the flow cell (assuming the outlet remains at atmospheric pressure). Effluent was ejected to a Petri dish (5) and subsequently discarded to waste. 


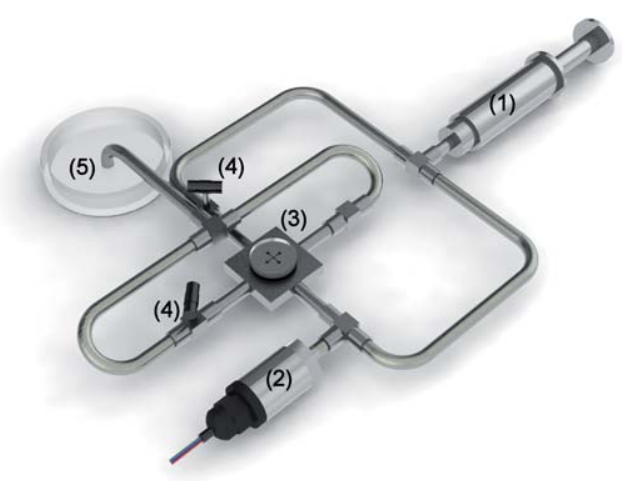

Fig. 2 Illustration of flow loop showing (1) stainless-steel syringe (driven by Harvard PHD-Ultra syringe pump), (2) Druck 5 psi gauge pressure sensor, (3) cross-slot flow-cell, (4) needle valves, (5) Petri dish to receive effluent.

\subsection{Extensional rates and dimensionless groups}

The Harvard PHD-Ultra syringe pump was used to deliver volume flow rates over the range $0.1 \mu \mathrm{L} \min ^{-1} \leq Q \leq 500$ $\mu \mathrm{L} \min ^{-1}$. This provided nominal extension rates at the stagnation point of $0.04 \mathrm{~s}^{-1} \leq \dot{\varepsilon}_{\text {nom }} \leq 200 \mathrm{~s}^{-1}$, calculated according to:

$$
\dot{\varepsilon}_{\text {nom }}=\frac{Q}{w^{2} d}
$$

This definition of $\dot{\varepsilon}_{\text {nom }}$ is based on the assumption that fluid at the stagnation point accelerates at a constant rate from zero up to the superficial flow velocity $(U=Q / 2 w d)$ at the slot entrance, a distance $w / 2$ from the stagnation point. This is likely to be reasonable only if the flow is plug-like within the channels. The validity of this assumption will be investigated by using flow velocimetry to directly measure the flow velocity between the channel entrances and experimentally determine the strain rate for both Newtonian and non-Newtonian fluids.

The Reynolds number for this flow-rate driven experiment is calculated using $R e=\rho U D_{h} / \eta(\dot{\gamma})$, where $D_{h}=2 w d /(w+d)$ is the hydraulic diameter, $\rho \approx 1 \mathrm{~g} \mathrm{~cm}^{-3}$ is the fluid density, and $\eta(\dot{\gamma})$ is the shear rate dependent shear viscosity determined from coneand-plate measurements (see Section 2.2). Within the cross-slot, assuming an ideal planar extensional flow, $u=\left[\dot{\varepsilon}_{\text {nom }} x,-\dot{\varepsilon}_{\text {nom }} y, 0\right]$ the appropriate value of the characteristic shear rate is $\dot{\gamma}=\sqrt{\frac{1}{2} \mathrm{II}(\dot{\gamma})}=2 \dot{\varepsilon}_{\text {nom }}$, where $\operatorname{II}(\dot{\gamma})$ is the second invariant of the shear rate tensor $\dot{\gamma}=\nabla \mathbf{u}+\nabla \mathbf{u}^{\mathbf{T}}$. For the range of volume flow rates investigated we obtain a range of $10^{-8} \leq R e \leq 0.16$.

We use the Weissenberg number $(W i)$ to characterize elastic effects near the stagnation point. Here $W i$ is defined as the ratio of fluid relaxation time $(\lambda)$ to characteristic flow time $\left(1 / \dot{\varepsilon}_{\text {nom }}\right)$. We use a relaxation time $\lambda_{M}=2.5 \mathrm{~s}$, determined from the linear viscoelasticity measurements described in Section 2.3, and the nominal value for the strain rate, $\dot{\varepsilon}_{\text {nom }}$, to evaluate $W i=\dot{\varepsilon}_{\text {nom }} \lambda_{M}$, which spans an experimental range of values $0.1 \leq W i \leq 500$.

The elasticity number $(E l)$ is defined as $E l=W i / R e$ and provides a measure of the relative importance of elastic and inertial effects in the flow field. In the present work we span the range $2600 \leq E l \leq 10^{8}$, indicating that elastic effects always dominate over inertial effects. Thus, our experimental geometry and test fluid permits us to examine stagnation point extensional flow effects in the almost complete absence of inertia.

\subsection{Test fluid preparation and rheological characterization}

The test fluid used in this study was an aqueous solution of 100 $\mathrm{mM} \mathrm{CPyCl}$ and $60 \mathrm{mM} \mathrm{NaSal}$ dissolved in deionised water. Both the $\mathrm{CPyCl}$ and the NaSal were supplied by Alfa Aesar. This particular surfactant/counterion system has been discussed at length by Rehage and Hoffman ${ }^{4,54}$ and has previously been studied in microfluidic shear ${ }^{55}$ and cross-slot extensional flows. ${ }^{23}$ The $\mathrm{CPyCl}$ and $\mathrm{NaSal}$ powders were weighed and added to the appropriate volume of deionised water. The mixture was stirred vigorously for three days and then left to equilibrate at room temperature in dry, unlit conditions for a further ten days before any experiments were conducted.

The storage and loss moduli, $G^{\prime}(\omega)$ and $G^{\prime \prime}(\omega)$ of the test fluid were measured at $22^{\circ} \mathrm{C}$ (close to ambient laboratory temperature at which cross-slot experiments were performed) using a TA Instruments AR-G2 stress-controlled rheometer with a stainless steel $40 \mathrm{~mm}$ diameter $2^{\circ}$ cone-and-plate. The results are presented in Fig. 3a, and have been fitted with a single mode Maxwell model, given in eqn (2): ${ }^{1}$

$$
G^{\prime}(\omega)=\frac{G_{0}\left(\lambda_{M} \omega\right)^{2}}{1+\left(\lambda_{M} \omega\right)^{2}}, G^{\prime \prime}(\omega)=\frac{G_{0} \lambda_{M} \omega}{1+\left(\lambda_{M} \omega\right)^{2}} .
$$

From the fit to the data, values for the Maxwell relaxation time $\left(\lambda_{M}=2.5 \mathrm{~s}\right)$ and plateau modulus $\left(G_{0}=32 \mathrm{~Pa}\right)$ were obtained. Following the methods described by Turner and Cates ${ }^{56}$ we also
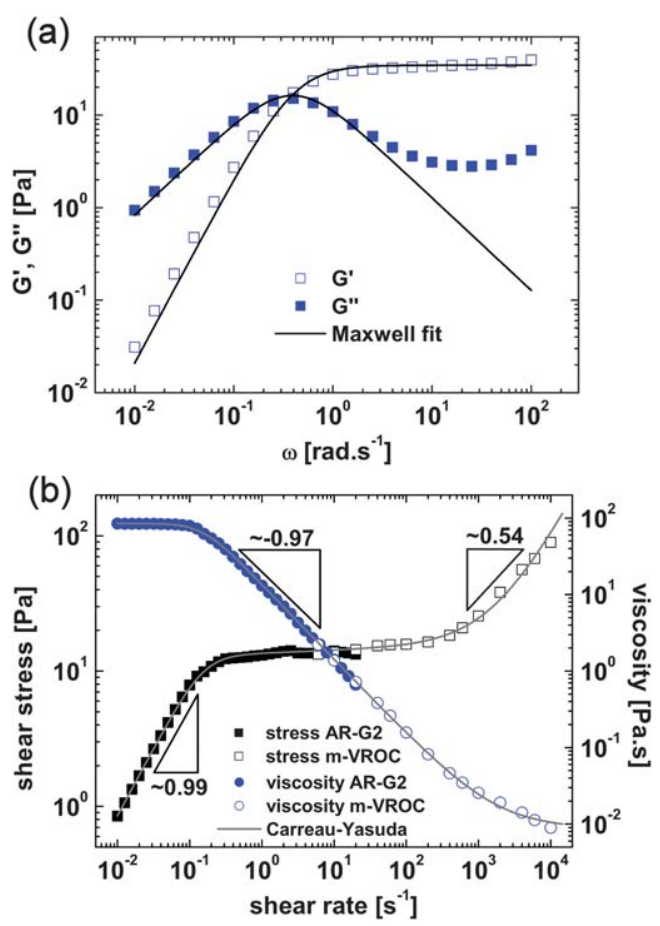

Fig. 3 Shear rheology of the $100 \mathrm{mM} \mathrm{CPyCl} / 60 \mathrm{mM}$ NaSal test fluid: (a) linear viscoelastic properties fitted with a single-mode Maxwell model. (b) Steady shear viscosity measured using cone-and-plate and m-VROC micro-capillary rheometry and fitted with a Carreau-Yasuda model. 
estimate the characteristic micellar breakage time $\left(\lambda_{\text {break }}=1.25 \mathrm{~s}\right)$ and reptation time $\left(\lambda_{\text {rep }}=5 \mathrm{~s}\right)$.

Fig. 3b shows the steady shear rheology of the test fluid. For shear rates $\dot{\gamma}<20 \mathrm{~s}^{-1}$ this data was measured using the AR-G2 cone-and-plate in the same configuration as previously described. To access higher shear rates, up to $\dot{\gamma} \approx 10^{4} \mathrm{~s}^{-1}$, a Rheosense m-VROC microfluidic rheometer was used. ${ }^{55,57}$ There is a good overlap between the data obtained by the two techniques. For $\dot{\gamma}<10^{-1} \mathrm{~s}^{-1}$ the fluid displays pseudo-Newtonian behaviour with an essentially invariant viscosity, $\eta_{0} \approx 85 \mathrm{~Pa}$.s. Above $\dot{\gamma} \approx 10^{-1} \mathrm{~s}^{-1}$ there is a pronounced stress plateau ( $\sigma_{\text {plateau }}$ $\sim 15 \mathrm{~Pa})$, which is indicative of shear banding. ${ }^{26,58,59}$ The stress plateau spans three decades of shear rate, during which the shear viscosity thins with a power-law index of $n \approx 0$, i.e. $\eta \propto \dot{\gamma}^{-1}$. For $\dot{\gamma}>500 \mathrm{~s}^{-1}$ the shear stress begins to increase again with shear rate, according to $\sigma \propto \dot{\gamma}^{0.54}$. In general these measurements are in excellent agreement with those made by previous authors with the same fluid..$^{55,59,60}$ The steady shear rheology is well described by the Carreau-Yasuda model ${ }^{61}$ (shown by the solid grey lines of Fig. 3b):

$$
\eta=\eta_{\infty}+\left(\eta_{0}-\eta_{\infty}\right)\left[1+\left(\dot{\gamma} / \dot{\gamma}^{*}\right)^{a}\right]^{(n-1) / a}
$$

where $\eta_{\infty}$ is the infinite-shear-rate viscosity ( $\left.=0.0085 \mathrm{~Pa} . \mathrm{s}\right), \eta_{0}$ is the zero-shear-rate viscosity (=85 Pa.s), $\dot{\gamma}^{*}$ is the characteristic shear rate for the onset of shear-thinning $\left(=0.15 \mathrm{~s}^{-1}\right), n$ is the "power-law exponent" $(=0.02)$ and $a$ is a dimensionless fitting parameter $(=2.5)$ that influences the speed of the transition from constant shear viscosity to the power-law region. We note that this generalized Newtonian fluid (GNF) model accurately describes the shear-thinning behaviour, but does not account for viscoelasticity, therefore its applicability is restricted. Nevertheless, as will be shown, this simple model can be used to predict fully developed velocity profiles, for conditions where viscoelastic memory effects are not dominant.

\subsection{CaBER extensional rheometry}

To characterize the relaxation time and extensional viscosity of the $100 \mathrm{mM} \mathrm{CPyCl} / 60 \mathrm{mM} \mathrm{NaSal}$ test fluid in an extensional flow, capillary breakup extensional rheometry (CaBER) was used. ${ }^{62,63}$ The device holds a discrete volume of fluid between parallel plates of diameter $D_{0}=6 \mathrm{~mm}$ and initial separation $L_{0}=1 \mathrm{~mm}$ (initial aspect ratio $\Lambda_{0}=L_{0} / D_{0}=0.167$ ). At time $t_{i}=-1 \mathrm{~s}$ the top endplate was displaced upwards following an exponential profile $L(t)=L_{0} \mathrm{e}^{\dot{\varepsilon}_{0} t}$ to achieve a final plate separation of $L_{f}=8 \mathrm{~mm}$ at time $t=0 \mathrm{~s}$ (final aspect ratio $\left.\Lambda_{f}=L_{f} / D_{0}=1.33\right)$. A relatively low stretching rate $\dot{\varepsilon}_{0}=\ln 8 \mathrm{~s}^{-1}$ was used because at higher rates the fluid thread tended to snap or to detach from one or other of the endplates, or elastic peeling instabilities were observed. ${ }^{64}$ The evolution of the liquid filament diameter $(D(t))$ was monitored at the midplane between the endplates (i.e. at $L=L_{f} / 2$ ) using a laser micrometre. The dynamics of the liquid bridge thinning and breakup process were also recorded at 60 frames per second using a 6 megapixel CCD camera (Casio Exilim EX-F1), at a resolution of $\sim 7 \mu \mathrm{m} /$ pixel. The filament diameter (normalized by $D_{0}$ ) as a function of time (normalized by the breakup time, $t_{c}$ ) for three individual CaBER experiments is shown in Fig. 4a along with snapshots of the
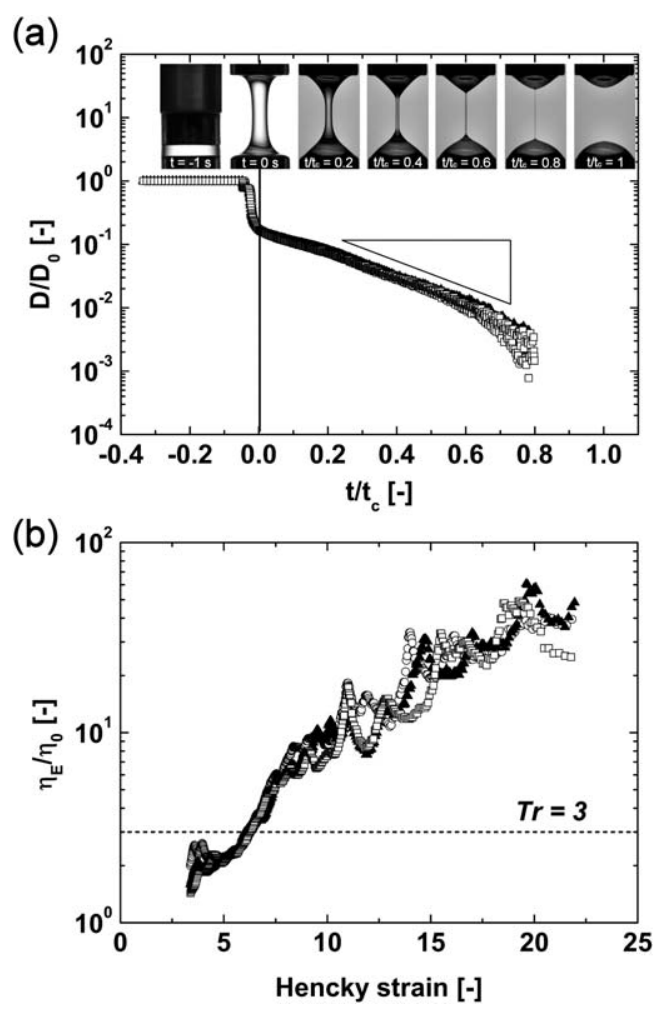

Fig. 4 Capillary breakup extensional rheometry (CaBER) of the $100 \mathrm{mM} \mathrm{CPyCl} / 60 \mathrm{mM}$ NaSal test fluid. (a) Filament diameter as a function of time. (b) Trouton ratio as a function of Hencky strain. The initial and final aspect ratios of the CaBER were $\Lambda_{0}=0.167$ and $\Lambda_{f}=$ 1.33 , respectively.

filament profile corresponding to one of the three experiments. The breakup times for the experiments were $t_{c}=25 \mathrm{~s}, 21 \mathrm{~s}$ and 21 s. For a model viscoelastic fluid in the elasto-capillary thinning regime the filament midplane diameter should decay exponentially as $D(t) \propto \exp \left(-t / 3 \lambda_{C a B E R}\right),{ }^{65}$ where $\lambda_{C a B E R}$ is the fluid relaxation time. Values of $\lambda_{C A B E R}$ determined from the three experiments shown in Fig. $4 \mathrm{a}$ were: $2.30 \mathrm{~s}, 1.83 \mathrm{~s}$ and $1.93 \mathrm{~s}$, giving a mean value ( \pm one standard deviation) of $\lambda_{\text {CaBER }}=2.02$ $\pm 0.25 \mathrm{~s}$, close to $\lambda_{M}$, as expected for this final aspect ratio, $\Lambda_{f}=$ $1.33 .^{63}$

The transient extensional strain rate $\dot{\varepsilon}(t)$ in the thinning fluid filament can be determined from the rate of decay of the filament diameter according to $\dot{\varepsilon}(t)=-(2 / D(t)) .(d D(t) / d t) .{ }^{62,65}$ This can be integrated with respect to time to give the instantaneous accumulated Hencky strain, $\varepsilon(t)=\int_{0}^{t} \dot{\varepsilon} d t=2 \ln \left(D_{0} / D(t)\right)$. Assuming elastic forces in the thinning fluid filament balance surface tension forces, the apparent transient extensional viscosity $\eta_{E}(t)$ of the fluid can also be determined from the measurement of $D(t)$ according to $\eta_{E}(t) \approx-\gamma_{L V} /(d D(t) / d t),{ }^{65}$ where $\gamma_{L V}$ is the surface tension, taken to be $32 \mathrm{mN} \mathrm{m}^{-1}$, as reported by Akers and Belmonte. ${ }^{66}$ In Fig. $4 \mathrm{~b}$ we show the measured Trouton ratio, $\mathrm{Tr}=$ $\eta_{E}(t) / \eta_{0}$, as a function of the Hencky strain determined from the data in Fig. 4a. The Trouton ratio increases up to a value of $T r \approx$ 40 at high strains, well above the Newtonian limit of $\operatorname{Tr}=3$, showing that the fluid is quite significantly strain hardening in a transient extensional flow. 


\subsection{Micro-particle image velocimetry}

Micro-particle image velocimetry ( $\mu$-PIV) was performed on test fluid seeded with $1.1 \mu \mathrm{m}$ diameter fluorescent tracer particles (Nile Red, Molecular Probes, Invitrogen; Ex/Em: 520/580 nm; $c_{p}$ $\approx 0.02 \mathrm{wt} . \%$ ). The imaging system consisted of a 1.4 megapixel, frame-straddling CCD camera (TSI Instruments, PIV-Cam) and an inverted microscope (Nikon Eclipse TE 2000-S). A $10 \times 0.25$ NA objective was used to focus on the mid-plane of the cross-slot flow cell. The resulting measurement depth $\left(\delta z_{m}\right)$ over which microparticles contribute to the determination of the velocity field is $\delta z_{m} \approx 50 \mu \mathrm{m},{ }^{67}$ or $\sim 5 \%$ of the depth of the flow cell, $d$. The fluid was illuminated by a double-pulsed $532 \mathrm{~nm} \mathrm{Nd:YAG} \mathrm{laser}$ with pulse width $\delta t=5 \mathrm{~ns}$. The fluorescent seed particles absorb the laser light and emit at a higher frequency. The laser light is filtered out with a G-2A epifluorescent filter, so that only the fluorescing particles are seen by the CCD array. Images were captured in pairs with a time separation $(1.2 \mu \mathrm{s}<\Delta t<60000 \mu \mathrm{s})$ chosen to achieve an average particle displacement of $\sim 4$ pixels, optimal for subsequent analysis. Image pairs were captured at a rate of approximately four pairs per second. The standard cross-correlation PIV algorithm (TSI insight software), with interrogation areas of $16 \times 16$ pixels and Nyquist criterion, was used to analyze each image pair. For steady flows, twenty image pairs were captured and ensemble-averaged in order to obtain full-field velocity maps in the vicinity of the stagnation point. For unsteady flows only five successive image pairs were averaged.

\subsection{Birefringence measurement}

Measurements of flow-induced birefringence in complex fluids can provide a measure of the segmental anisotropy of macromolecules as they deform in a flow field. In systems where the macromolecular parameters are well known and defined, such measurements can be compared with models in order to estimate the degree of accumulated macromolecular strain. ${ }^{68}$ Over a limited range of molecular deformations, many systems of flexible macromolecules obey the stress-optical rule (SOR), which states that the magnitude of the birefringence $(\Delta n)$ is directly proportional to the principal stress difference $\left(\Delta \sigma=\sigma_{x x}\right.$ $\left.-\sigma_{y y}\right):{ }^{69}$

$$
\Delta n=C \Delta \sigma
$$

where the constant of proportionality, $C$, is called the stressoptical coefficient. In this case, by measuring $\Delta n$ at the stagnation point, a value for the apparent extensional viscosity can be estimated in the cross-slot using $\eta_{\mathrm{E}, \mathrm{app}}=\Delta \sigma / \dot{\varepsilon}_{\text {nom }}$. The value of $C$ for the $100 \mathrm{mM} \mathrm{CPyCl} / 60 \mathrm{mM}$ NaSal system has been determined by Ober et al. ${ }^{55}$ to be $C=-1.1 \times 10^{-7} \mathrm{~Pa}^{-1}$, in close agreement with the value reported by $\mathrm{Hu}$ and $\mathrm{Lips}^{70}$ with a similar system. In their cross-slot extensional flow experiments, Pathak and Hudson ${ }^{23}$ reported that the SOR remained valid for $100 \mathrm{mM} \mathrm{CPyCl} / 60 \mathrm{mM} \mathrm{NaSal}$ up to $W i \approx 0.8$.

We measured the spatial distribution of flow-induced birefringence in the vicinity of the stagnation point using an ABRIO birefringence microscope system (CRi, Inc.), which has been described in detail by Shribak and Oldenbourg ${ }^{71}$ and in a previous publication by Ober et al. ${ }^{55}$ Briefly, the cross-slot flow cell was placed on the imaging stage of an inverted microscope
(Nikon Eclipse TE 2000-S). Using a $20 \times 0.5$ NA objective, first the bottom surface and then the top surface of the $1 \mathrm{~mm}$ deep flow cell were brought into focus in order to precisely determine the position of the mid-plane of the flow cell, which was subsequently used as the measurement plane. The ABRIO system passes circularly polarized monochromatic light (wavelength 546 $\mathrm{nm}$ ) first through the sample, then through a liquid crystal compensator optic and finally onto a CCD array. Optical anisotropy in the fluid can be viewed in real-time on a PC monitor. When an image is captured the CCD records five individual frames with the liquid crystal compensator configured in a specific polarization state for each frame. Using data processing algorithms described by Shribak and Oldenbourg, ${ }^{71}$ the ABRIO software converts the five individual frames into a single full-field map of retardation and orientation angle. The system can measure the retardation $(R)$ to a nominal accuracy of $\sim 0.02$ $\mathrm{nm}$, and has an excellent spatial resolution (projected pixel size $\sim 0.5 \mu \mathrm{m}$ with a $20 \times$ objective lens). The relationship between retardation and birefringence is given by $R=d \Delta n$, where $d$ is the depth of the flow cell.

\subsection{Pressure drop and viscometry}

The pressure difference arising from viscous flow in the cross-slot geometry is measured across an inlet and an outlet channel using a Druck 5 psi pressure sensor, labelled (2) in Fig. 2. By closing the two needle valves, labelled (4), the pressure drop can be measured for steady viscous flow of fluid around a corner of the cross-slot $\left(\Delta P_{\text {shear }}\right)$ enabling an estimate of the shear viscosity of the fluid, $\eta$. Assuming Poiseuille flow in a rectangular channel of total length $2 l$ and neglecting perturbations arising from the presence of the corner of the cross, ${ }^{72}$ the wall shear rate $\left(\dot{\gamma}_{\text {wall }}\right)$ and shear stress $\left(\sigma_{\text {wall }}\right)$ can be found from eqn (5) and (6), respectively, assuming a $2 \mathrm{D}$ flow:

$$
\begin{gathered}
\dot{\gamma}_{\text {wall }}=\frac{6 Q}{w^{2} d} \\
\sigma_{\text {wall }}=\frac{-\Delta P_{\text {shear }}}{2 l} \frac{w}{2}
\end{gathered}
$$

The shear viscosity $(\eta)$ follows:

$$
\eta=\frac{\sigma_{\text {wall }}}{\dot{\gamma}_{\text {wall }}} K
$$

where $K$ is a correction factor to account for the finite depth of the channel, given by: ${ }^{73}$

$$
K=1-\frac{192 w}{\pi^{5} d} \sum_{i=1,3,5, \ldots}^{\infty} \frac{\tanh (i \pi d / 2 w)}{i^{5}}
$$

leading to a correction factor $K \approx 0.88$, illustrating that assuming a $2 \mathrm{D}$ flow approximation results in approximately $12 \%$ error for the current channel aspect ratio. Eqn (6) can be easily generalized for a rectangular channel, resulting in $\sigma_{\text {wall }}=\frac{-\Delta P_{\text {shear }}}{2 l} \frac{w}{2} \frac{d}{w+d}$. Therefore the 2D flow approximation leads to an error of $16 \%$ in $\sigma_{\text {wall }}$ (in excess) and consequently we can estimate an error of $4 \%$ (also in excess) in the value of $\dot{\gamma}_{\text {wall }}$ predicted for Newtonian fluids assuming a 2D flow approximation. 
To improve the accuracy of this measurement for non-Newtonian fluids the Weissenberg-Rabinowitsch-Mooney correction can be applied to determine the true wall shear rate in the channel: ${ }^{57}$

$$
\dot{\gamma}_{\text {wall,true }}=\frac{\dot{\gamma}_{\text {wall }}}{3}\left(2+\frac{d \ln \dot{\gamma}_{\text {wall }}}{d \ln \sigma_{\text {wall }}}\right)
$$

However, although such an approach can give a good approximation to the shear viscosity of complex fluids when applied to steady flow around a corner, ${ }^{50} \eta$ can be determined more reliably using conventional (i.e. cone-and-plate) rheological methods, or by using a rectilinear microchannel flow such as the m-VROC, see Section 2.3. The main reason for measuring $\Delta P_{\text {shear }}$ in situ within the cross-slot geometry is so that it can be subtracted from the pressure drop measured with the two needle valves open $\left(\Delta P_{\text {total }}\right)$. This allows determination of the excess pressure drop ( $\left.\Delta P_{\text {excess }}\right)$ due to the extensional component in the flow-field. Assuming $\Delta P_{\text {excess }} \propto \Delta \sigma$, where $\Delta \sigma$ is the extensional stress in the elongating fluid elements, we can obtain a measure of the apparent extensional viscosity of the fluid:

$$
\eta_{E, \text { app }}\left(\dot{\varepsilon}_{\text {nom }}\right) \approx \frac{\Delta P_{\text {exces }}}{\dot{\varepsilon}_{\text {nom }}}=\frac{\Delta P_{\text {total }}-\Delta P_{\text {shear }}}{\dot{\varepsilon}_{\text {nom }}}
$$

The Trouton ratio of the fluid is then calculated according to $\operatorname{Tr}\left(\dot{\varepsilon}_{\text {nom }}\right)=\eta_{\mathrm{E}, \text { app }}\left(\dot{\varepsilon}_{\text {nom }}\right) / \eta(\dot{\gamma})$, where $\eta(\dot{\gamma})$ is the measured value of the shear viscosity (from cone-and-plate data) and the appropriate value of $\dot{\gamma}$ in the cross-slot is taken to be $\dot{\gamma}=2 \dot{\varepsilon}_{\text {nom }}$, assuming ideal planar elongation. The expected Trouton ratio for Newtonian fluids undergoing planar elongational flow is $\operatorname{Tr}=4 .^{74}$

\subsection{Numerical method}

In addition to the experimental measurements, 3D-simulations of the steady viscous flow in the cross-slot were performed to serve as a reference for validation in the Newtonian fluid flow case. We use a computational fluid dynamics code based on the finite volume methodology $(\mathrm{FVM})^{75,76}$ to solve the equations of conservation of mass and momentum assuming isothermal, incompressible flow:

$$
\begin{gathered}
\boldsymbol{\nabla} \cdot \mathbf{u}=0 \\
\rho\left[\frac{\partial \mathbf{u}}{\partial t}+(\mathbf{u} \cdot \nabla) \mathbf{u}\right]=-\nabla p+\nabla \cdot \tau
\end{gathered}
$$

where $\rho$ is the density of the fluid, $t$ is the time, $\mathbf{u}$ is the velocity vector, $p$ is the pressure and $\tau$ is the extra-stress tensor, which in the case of Newtonian, or GNF, fluids is given as:

$$
\tau=\tau_{s}=\eta\left(\nabla \mathbf{u}+\nabla \mathbf{u}^{\mathrm{T}}\right)
$$

where $\eta$ is the shear viscosity of the fluid. The numerical code used here was developed to work with a number of viscoelastic rheological models, and therefore the constitutive equation, even for the case of a Newtonian fluid flow, is solved separately from the momentum equation, despite being explicit in the Newtonian case. This separation of the equations has been used extensively with Newtonian fluid flows ${ }^{75-78}$ and it has been shown to have no impact on the calculations except for the additional memory used to allocate the extra-stress tensor. ${ }^{75,76}$
In the present formulation, calculations are performed using a fully-implicit, time-marching pressure-correction algorithm in which the resulting algebraic equations relate the dependent variables, evaluated at the centre of the control volumes $(\mathrm{CV})$ forming the computational mesh, to the values in the nearby surrounding CV. For the discretization of the equations, central differences are used for the diffusive terms, and the CUBISTA high-resolution scheme ${ }^{79}$ is used to discretize the advective terms of the momentum equation. An implicit first-order Euler scheme is used for the time derivative discretization, but under steady flow conditions this term vanishes and therefore, the numerical method has second-order accuracy in steady flow simulations.

The characteristics of the geometry and the physical properties of the fluid used in the numerical calculations were selected to match those measured experimentally. Furthermore, the meshes used to map the computational domain are block-structured, orthogonal and non-uniform, with minimum cell sizes $\delta x_{\min }=$ $\delta y_{\min }=\delta z_{\min }=0.04 \mathrm{w}$. Computations with a more refined mesh $\left(\delta x_{\min }=\delta y_{\min }=\delta z_{\min }=0.02 \mathrm{w}\right)$ led to similar results (maximum variation below $1 \%$ in the velocity profiles), therefore the accuracy of the numerical simulations is high. In terms of boundary conditions, we impose no-slip conditions at the walls, constant velocity profiles at the inlet, and vanishing stream wise gradients at the outlets for all computed variables, except for pressure which is linearly extrapolated from the two adjacent upstream cells.

\section{Results and discussion}

\subsection{Experimental validation: Newtonian flow in the cross-slot microchannel}

3.1.1. Flow velocimetry. Before conducting experiments with the complex wormlike micellar fluid, which we believe likely to exhibit elastic flow instabilities at low $R e,{ }^{23}$ it is important to confirm the symmetry and stability of the flow field in the Newtonian case. Accordingly, $\mu$-PIV experiments were performed in the microfluidic cross-slot geometry over a wide range of flow rates using water as a representative Newtonian working fluid. The results are summarized in Fig. 5, including comparison with numerical simulations.

In Fig. 5a we show an example of the velocity field in the crossslot geometry for the flow of water at a volumetric flow rate of $1600 \mu \mathrm{L} \mathrm{min}{ }^{-1}$, or $R e \approx 20$, which is more than two orders of magnitude greater than the highest $R e$ reached in subsequent experiments with $\mathrm{CPyCl}$ solution. Qualitatively, the Newtonian flow field displays a high degree of symmetry about the $x$ and $y$ axes, with the stagnation point located at the origin of coordinates in the centre of the cross. Velocity profiles taken across the inlet and outlet channels ( $\left.v_{x} v s . y, v_{y} v s . x\right)$ at various distances up and down stream from the stagnation point closely match the parabolic numerical solution for Poiseuille flow of a Newtonian fluid in a rectangular channel at this volume flow rate (Fig. 5b). Experimental velocity profiles taken along the $x$ and $y$ axes $\left(v_{x} v s\right.$. $x, v_{y}$ vs. $y$ ) are shown in Fig. 5c, together with the corresponding numerical predictions, confirming the symmetry of the velocity field around the stagnation point at the centre of the geometry. Differentiating the curves in Fig. 5c gives the centre-line strain rate as a function of position shown in Fig. 5d. Here the vertical 

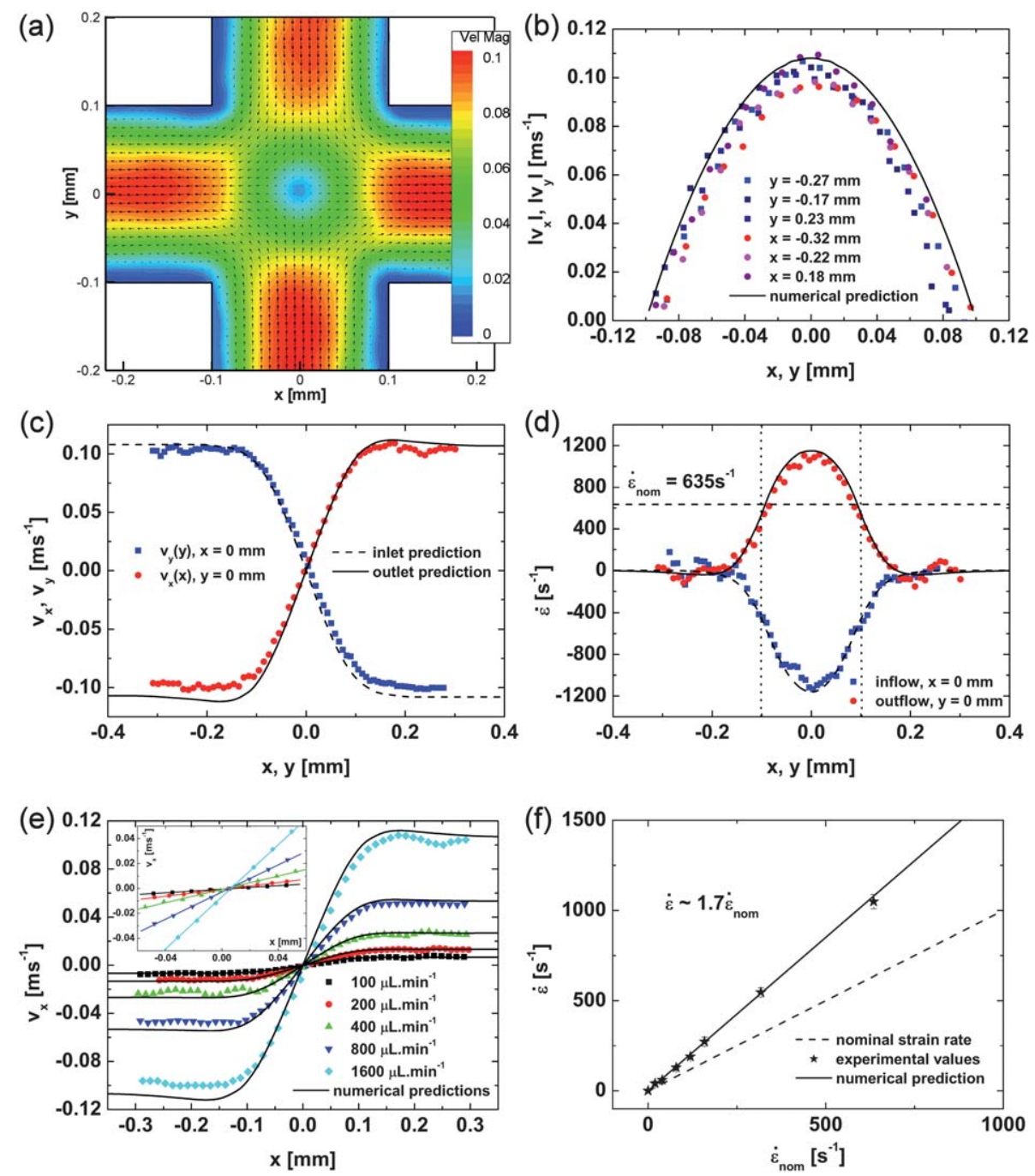

Fig. 5 (a) Velocity field in the vicinity of the stagnation point for water flowing at $Q=1600 \mu \mathrm{L} \mathrm{min}{ }^{-1}\left(U=63.5 \mathrm{mms}^{-1}, \dot{\varepsilon}_{n o m}=635 \mathrm{~s}^{-1}, R e \approx 20\right)$, colour scale in $\mathrm{ms}^{-1}$. (b) Parabolic velocity profiles across inlet and outlet channels, under the same conditions as (a), showing close agreement with the numerical solution for a Newtonian fluid. (c) Streamwise flow velocity as a function of position along the symmetry axes of the cross-slot under the same conditions as (a). (d) Strain-rate as a function of position along the symmetry axes of the cross-slot, determined by differentiating the data in (c). The dotted lines represent the channel entrances and the dashed line shows the nominal strain rate. (e) Velocity as a function of position along outflow symmetry axis $(y=0 \mathrm{~mm}$ ) for a range of $Q$ values (inset shows linear fits to the data between $x \sim \pm 0.05 \mathrm{~mm}$, from which the strain rate is determined). (f) Measured and predicted variation in the strain-rate as a function of nominal strain rate for water in the cross-slot.

dotted lines represent the position of the channel entrances at $x$, $y= \pm 100 \mu \mathrm{m}$ and the horizontal dashed line shows the value of the nominal strain rate at this volume flow rate, calculated using eqn (1). We observe a compressive strain rate along the inbound stagnation point streamline and an essentially equal and opposite extensional strain rate along the outbound streamline. The extension rate has a maximum value at the stagnation point and, within noise, decays to zero within approximately $100 \mu \mathrm{m}$ inside the channel entrance. Again, the experimental data closely match the numerical solutions for Newtonian flow. The peak extension rate at the stagnation point is significantly higher than the expected nominal value. Fig. 5e shows the flow velocity along the outbound stagnation point streamline, measured over a range of volume flow rates. The inset of Fig. 5e shows detail of the flow velocity profile over a distance of approximately $\pm 50 \mu \mathrm{m}$ either side of the stagnation point. Over this distance the strain rate $(\dot{\varepsilon})$ is approximately constant, as shown by the straight-line fits to the data, the gradient of which give an average value for $\langle\dot{\varepsilon}\rangle$. Fig. 5f shows $\langle\dot{\varepsilon}\rangle$ (measured as described) as a function of the expected nominal value, $\dot{\varepsilon}_{\text {nom }}$, from eqn (1). We find that the measured strain rate near the stagnation point varies linearly with the flow rate, but is higher than $\dot{\varepsilon}_{\text {nom }}$ by a constant factor of 1.7. This is consistent with the parabolic velocity profiles across the channels, which exhibit a maximum value of approximately $1.7 U$ at the channel centre-line, and also agrees very well with the numerical simulations.

3.1.2. Pressure drop and viscometry. Due to the very low Trouton ratio of Newtonian fluids it is not viable to accurately isolate an excess pressure drop in extensional flow in our crossslot device. The apparent extensional viscosity of Newtonian fluids therefore appears to be below the experimental noise floor 
in these initial calibration experiments. However, the pressure sensor was tested by measuring $\Delta P_{\text {shear }}$ and finding a value for the solvent viscocity, $\eta_{S}$, for water using eqn (5)-(7). Fig. $6 a$ shows the pressure measured as a function of time as the flow rate was incremented from $0 \mu \mathrm{L} \mathrm{min}{ }^{-1}$ up to $5000 \mu \mathrm{L} \mathrm{min}^{-1}$ in ten steps of four second intervals. The rise time to steady pressure at each increment in flow rate is virtually instantaneous $(\delta t<250$ $\mathrm{ms}$ ), and when the flow stops at the end of the test the pressure quickly returns to the baseline level. At each incremental flow rate, the plateau pressure drop data can be averaged and plotted as a function of superficial flow velocity, $U$. As shown in Fig. 6b, for $U<0.15 \mathrm{~ms}^{-1}$ the pressure drop increases linearly and with a similar gradient to that expected based on the viscosity of water at the ambient laboratory temperature $\left(22 \pm 1{ }^{\circ} \mathrm{C}\right)$. The wall stress according to eqn (5) is plotted against the wall shear rate according to eqn (6) in the inset of Fig. 6b. The gradient of a straight-line fit through the origin gives a value of $\eta_{S}=0.90 \pm$ $0.02 \mathrm{mPa} . \mathrm{s}$, in reasonable agreement with the value of $\eta_{S}=0.97$
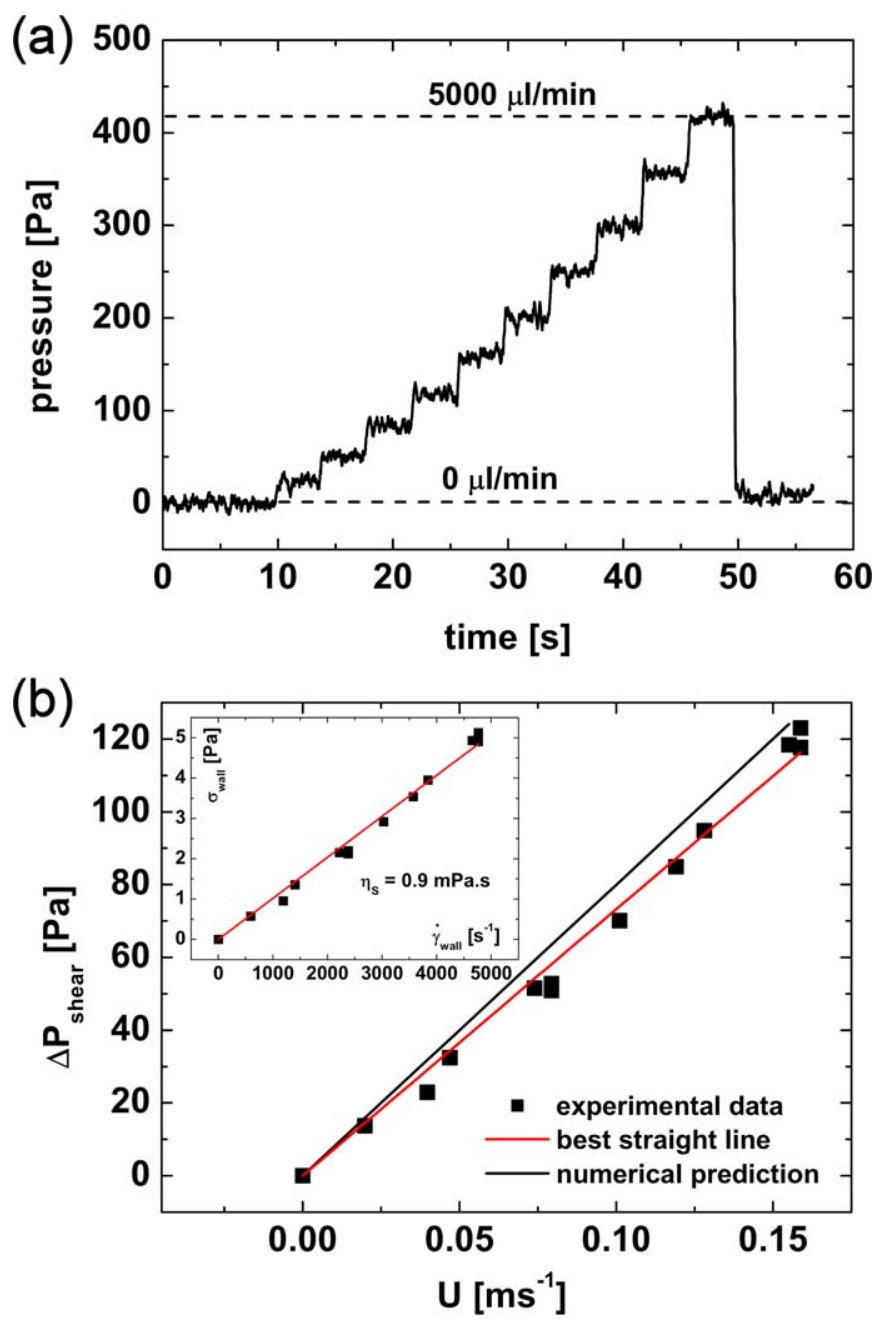

Fig. 6 (a) Pressure drop as a function of time for the flow of water around the corner of the cross-slot in ten increments of $500 \mu \mathrm{L} \mathrm{min}{ }^{-1}$ taken at 4 s intervals. (b) Pressure drop around the corner as a function of superficial flow velocity, determined from data such as (a). Inset shows a linear relationship between wall stress and shear rate, from which the viscosity of water can be estimated at $\eta_{S} \approx 0.9 \mathrm{mPa}$ at $22 \pm 1^{\circ} \mathrm{C}$, close to the expected value.
$\mathrm{mPa} . \mathrm{s}$ obtained from the numerical simulations and $\eta_{S}=0.955$ $\mathrm{mPa}$.s obtained from published tables. ${ }^{80}$

\subsection{Flow of $100 \mathrm{mM} \mathrm{CPyCl} / 60 \mathrm{mM}$ NaSal solution}

3.2.1. Flow velocimetry. Having established confidence in the symmetry, stability and accuracy (compared with numerical predictions) of the stagnation point flow field with a Newtonian fluid in the cross-slot geometry, we proceed to use $\mu$-PIV to quantify the flow field for the $100 \mathrm{mM} \mathrm{CPyCl} / 60 \mathrm{mM} \mathrm{NaSal}$ wormlike micellar test solution.

Transition from symmetric to steady asymmetric flow. In Fig. 7 we present the PIV vector velocity fields obtained with the $\mathrm{CPyCl} / \mathrm{NaSal}$ solution over a range of volumetric flow rates 0.8

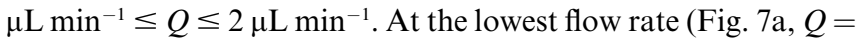
$0.8 \mu \mathrm{L} \mathrm{min}{ }^{-1}, \dot{\varepsilon}_{\text {nom }}=0.32 \mathrm{~s}^{-1}, W i=0.8$ ) the flow field appears essentially Newtonian-like, with a high degree of symmetry about a centrally located, circular stagnation point (see also the streakline imaging presented in Movie SI $1 \uparrow$ in the Supplementary Information). On increasing the flow rate to $Q=1.2 \mu \mathrm{L} \mathrm{min}{ }^{-1}$ $\left(\dot{\varepsilon}_{\text {nom }}=0.48 \mathrm{~s}^{-1}, W i=1.2\right.$, Fig. $\left.7 \mathrm{~b}\right)$ the contour plot of the velocity magnitude near the stagnation point takes on an elliptical shape with the major and minor axes oriented at approximately $\pm 45^{\circ}$ to the outflow direction. In Fig. 7c ( $Q=1.6 \mu \mathrm{L}$ $\left.\min ^{-1}, \dot{\varepsilon}_{\text {nom }}=0.64 \mathrm{~s}^{-1}, W i=1.6\right)$ the elliptical shape of the stagnation point has become accentuated and it is clear that the flow has become asymmetric, with a significant fraction of the fluid entering from the top/bottom channels being directed preferentially towards the right/left exit channels, respectively (note that the velocity vectors along the horizontal centre-line, $y=0$, are no longer horizontal). This is equivalent to the flow bifurcation observed by Pathak and Hudson in wormlike micellar solutions. ${ }^{23}$ At these flow rates, the flow remains steady and the flow asymmetry can occur in either direction with respect to the $x, y$ axes, and thus appears qualitatively similar to the supercritical pitchfork bifurcation reported by Arratia et al. ${ }^{42}$ and modelled by Poole et al. $^{44}$ and Rocha et $a{ }^{45}$ It should be noted that the bifurcation we report is purely elastic in origin; the Reynolds number in Fig. 7 is $R e<10^{-5}$. Streakline imaging of the flow in this steady bifurcated regime is provided in Movie SI2 in the Supplementary Information. $\dagger$ On further increasing the flow rate to $Q=2.0 \mu \mathrm{L} \min ^{-1}$ ( $\dot{\varepsilon}_{\text {nom }}=0.8 \mathrm{~s}^{-1}, W i=2$, Fig. $\left.7 \mathrm{~d}\right)$ the asymmetry is complete in the sense that $\sim 100 \%$ of the flow entering from the top channel exits via the right channel and $\sim 100 \%$ of the flow entering from the bottom channel exits via the left channel (see also the streakline imaging provided in Movie SI3 in the Supplementary Information $\dagger$ ). Such completely asymmetric flow was not reported in the cross-slot experiments of Pathak and Hudson ${ }^{23}$ and, to our knowledge, such a high degree of flow asymmetry has not been reported previously in a cross-slot flow. However, this flow is reminiscent of the bistable microfluidic "flip-flop" reported by Groisman et al. ${ }^{47}$ with an elastic, semi-dilute, high molecular weight polyacrylamide solution. In their case the direction of the bifurcation appeared to be determined by the presence of an asymmetric vortex in one of the entry channels, which were fabricated with different dimensions and geometric configurations. This is not true in the cross-slot; in our case both opposing entry channels have equal dimensions 

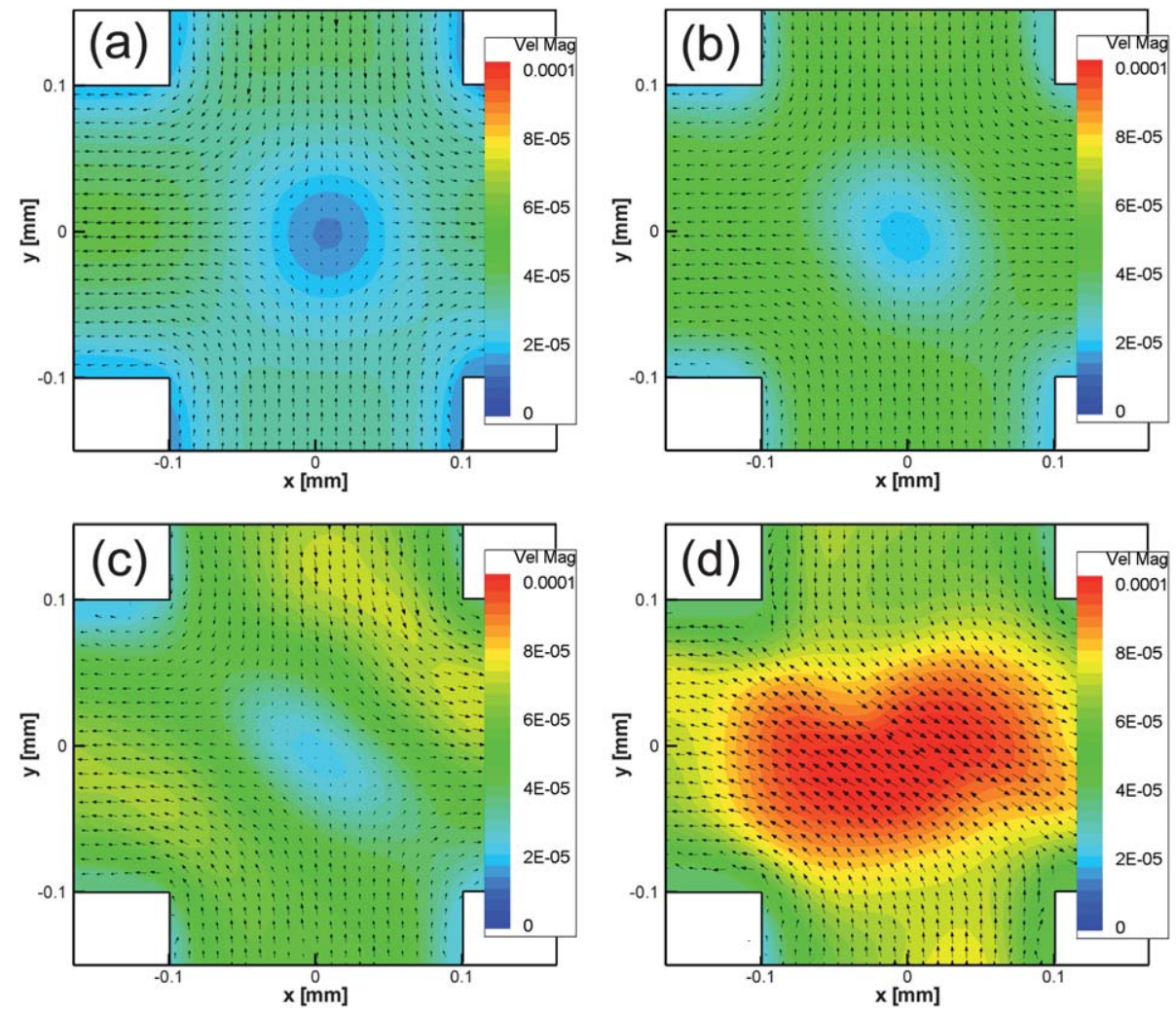

Fig. 7 Velocity field in the vicinity of the stagnation point for $100 \mathrm{mM} \mathrm{CPyCl} / 60 \mathrm{mM} \mathrm{NaSal}$ in cross-slot flow: (a) $Q=0.8 \mu \mathrm{L} \mathrm{min}{ }^{-1}, W i=0.8$ (b) $Q=$ $1.2 \mu \mathrm{L} \mathrm{min}{ }^{-1}, W i=1.2$ (c) $Q=1.6 \mu \mathrm{L} \mathrm{min}{ }^{-1}, W i=1.6$ (d) $Q=2.0 \mu \mathrm{L} \mathrm{min}^{-1}, W i=2$. The Reynolds number $R e<10^{-5}$ in all four cases. Colour scale bars represent the velocity magnitude and are in $\mathrm{ms}^{-1}$.

and the flow is fully developed by the time fluid elements reach the stagnation point region. In the cross-slot geometry we observe symmetry-breaking flow bifurcations in either direction at random, which are determined by random perturbations as the flow develops from start-up.

When the flow is symmetric, i.e. below $W i \approx 1$, velocity profiles taken across the inlet and outlet channels show a progression towards a more plug-like flow as the flow rate is increased, see Fig. 8a. The experimental data compare well with the predictions of the Carreau-Yasuda model (eqn (3)), as shown by the solid lines, assuming fully developed $2 \mathrm{D}$ flow. The data can also be fitted with an ad-hoc power-law model of the form:

$$
v_{x, y}=\left(\frac{2 n+1}{n+1}\right) \times\left(1-|y, x|^{(n+1) / n}\right)
$$

where the first argument refers to the inflow and the second argument refers to the outflow profile. The power-law exponent $n$ varies with Weissenberg number and reflects the level of shearthinning in the fluid rheology. Such profiles are valid for powerlaw fluids in fully developed 2D flow, a reasonable approximation given the large aspect ratio of the channel and the fact that under fully-developed flow conditions only the viscous shear rheology is important, assuming normal stress effects are small. At very low volumetric flow rates, e.g. $0.3 \mu \mathrm{L} \mathrm{min}^{-1}$, the velocity profile across the channel is essentially Newtonian-like and parabolic with a power-law exponent, $n$ close to unity. Here $\dot{\varepsilon}_{\text {nom }}=0.12 \mathrm{~s}^{-1}, \dot{\gamma}=0.24 \mathrm{~s}^{-1}$, which is very close to the commencement of the stress plateau on the flow curve in Fig. 3a.

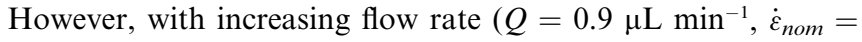
$0.36 \mathrm{~s}^{-1}, \dot{\gamma}=0.72 \mathrm{~s}^{-1}, W i=0.9$ ) the value of $n$ reduces to $n \approx 0.1$ and the flow profile becomes plug-like. High shear rate regions are localized close to the channel walls and a broad region with low shear rate fills the mid-section of the channel. Mair and Callaghan were the first to report that for pipe flow of a shear banding $\mathrm{CPyCl} / \mathrm{NaSal}$ solution the flow profile across the channel transitioned from Poiseuille-like to plug-like at shear rates corresponding to the onset of stress plateau region of the flow curve. ${ }^{81}$ Such behaviour has been confirmed recently in several studies of shear banding micellar fluids in rectilinear microchannels. ${ }^{55,82,83}$ Since the shear localization observed within the channel is closely related to the phenomenon of shear banding, for simplicity we will refer to it as such in the remainder of this article.

As was the case for the Newtonian fluid considered earlier, in the symmetric flow regime velocity profiles taken along the outflow axis (i.e. $v_{x}$ vs. $x$ along $y=0$ ) can be used to estimate the actual value of the strain rate near the stagnation point in the $\mathrm{CPyCl} / \mathrm{NaSal}$ test fluid. Examples of such profiles for various flow rates within the symmetric regime are shown in Fig. 8b, compared with the predictions of the Carreau-Yasuda model (eqn (3)). The inset of Fig. 8b shows linear regressions to the experimentally measured velocity between points approximately $50 \mu \mathrm{m}$ either side of the stagnation point. The gradients of these approximately linear sections provide an experimental measure of the strain rate near the stagnation point, which is shown for all the tested flow rates in Fig. 8c. For comparison, Fig. 8c also 

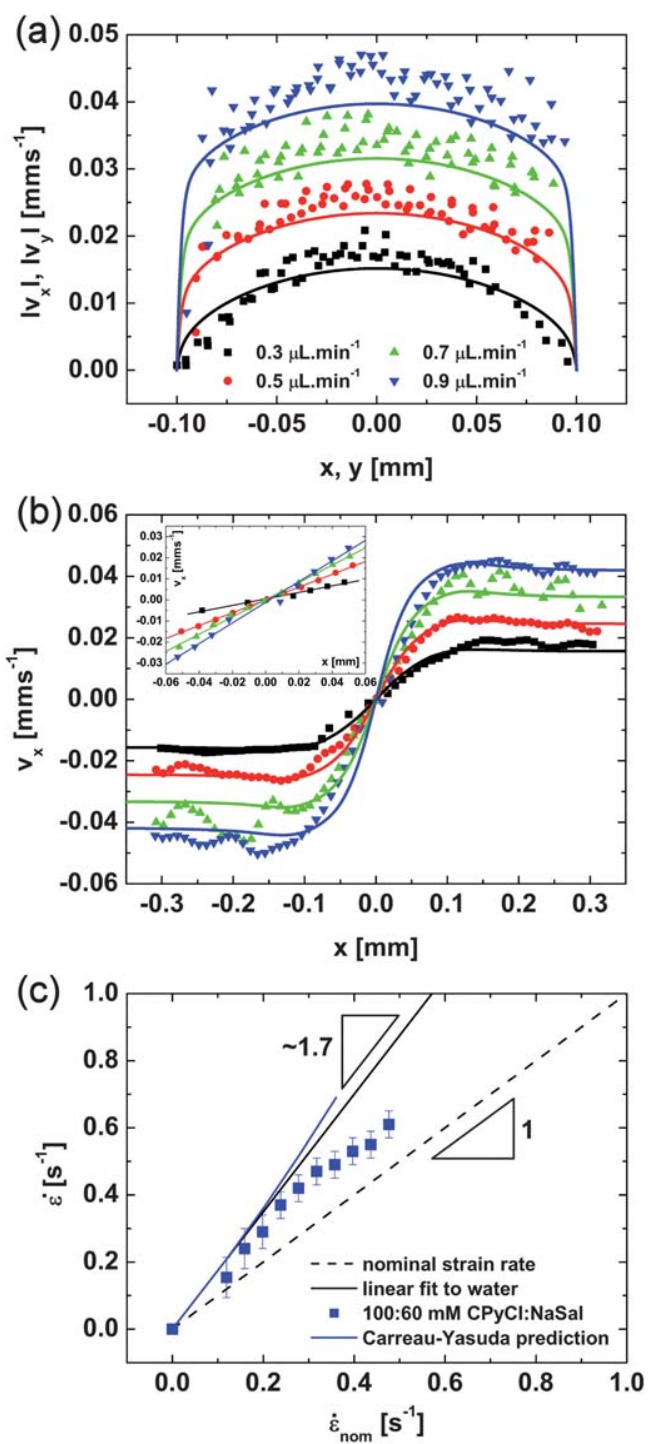

Fig. 8 (a) Velocity profiles across inlet and outlet channels of the crossslot for flow of $100 \mathrm{mM} \mathrm{CPyCl} / 60 \mathrm{mM} \mathrm{NaSal}$ at various values of $Q$ in the symmetric flow regime. Solid lines show the predictions of the CarreauYasuda model. (b) Velocity as a function of position along outflow symmetry axis ( $y=0 \mathrm{~mm}$ ) for a range of $Q$ values, where the solid lines show the predictions of the Carreau-Yasuda model. (Inset shows linear fits to the experimental data between $x \sim \pm 0.05 \mathrm{~mm}$, from which the strain rate is determined). (c) Strain rate as a function of the nominal strain rate for $100 \mathrm{mM} \mathrm{CPyCl} / 60 \mathrm{mM} \mathrm{NaSal}$ in cross-slot flow.

shows the strain rate measured and computed for water in the cross-slot and the numerically predicted evolution in the strain rate at the stagnation point for the Carreau-Yasuda fluid. The measured strain rates for the $\mathrm{CPyCl} / \mathrm{NaSal}$ solution at each flow rate lie between the expected nominal value, $\dot{\varepsilon}_{\text {nom }}$ given by eqn (1) and the values expected for a viscous Newtonian fluid. At very low flow rates the measured strain rate in the $\mathrm{CPyCl} / \mathrm{NaSal}$ solution is close to that of the Newtonian fluid, however as the flow rate increases, the measured strain rate near the stagnation point increases more slowly with $Q$ and approaches the nominal value. This is due to the development of increasingly plug-like flow characteristics in the channel, in agreement with the definition of $\dot{\varepsilon}_{\text {nom }}$ in eqn (1), which assumes a perfect plug flow. At
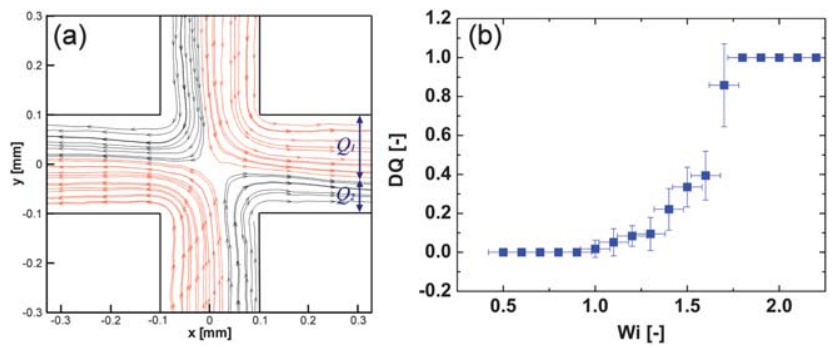

Fig. 9 (a) Experimentally determined streamlines for flow of $100 \mathrm{mM}$ $\mathrm{CPyCl} / 60 \mathrm{mM} \mathrm{NaSal}$ in the cross-slot during steady asymmetric flow at $Q=1.5 \mu \mathrm{L} \mathrm{min}{ }^{-1}\left(R e<10^{-5}, W i=1.5\right)$, in this instance corresponding to an asymmetry parameter of $D Q=\left|\left(Q_{1}-Q_{2}\right) /\left(Q_{1}+Q_{2}\right)\right| \approx 0.25$. (b) Evolution in the symmetry parameter as a function of $W i$, calculated from data such as shown in (a).

low strain rates, the Carreau-Yasuda model describes the experimental data reasonably well, closely following the Newtonian value. However at higher flow rates the Carreau-Yasuda model significantly over-predicts the strain rate. This is evident in Fig. $8 \mathrm{~b}$ where, between the channel entrances $(-0.1 \mathrm{~mm} \leq x \leq$ $0.1 \mathrm{~mm}$ ), the gradient of the Carreau-Yasuda model prediction becomes much steeper than the measured velocity profile as the flow rate increases.

In the bifurcated flow regime we have quantified the degree of flow asymmetry using an asymmetry parameter $(D Q)$, defined similarly to previous authors, ${ }^{44,45}$ except that we take the absolute value so that more experimental data can be averaged:

$$
D Q=\left|\frac{Q_{1}-Q_{2}}{Q_{1}+Q_{2}}\right|
$$

where $Q_{1}$ describes the amount of fluid that enters/exits via one entry/exit channel and $Q_{2}$ describes the amount that enters/exits via the opposite channel. For completely symmetric flow $D Q=$ 0 and for completely asymmetric flow $D Q=1$. Since the flow has been shown to become increasingly plug-like, the values of $Q_{1}$ and $Q_{2}$ can be estimated simply by measuring the separation of streamlines within the entry and exit channels, as shown in the example in Fig. 9a. From each image at each flow rate, four values of $Q_{1}$ and $Q_{2}$ are obtained (one in each channel) allowing calculation of an average and standard deviation for $D Q$. The evolution in this asymmetry parameter, $D Q$, as a function of $W i$ is shown in Fig. 9b, where the data points are the combined averages and standard deviations over three $\mu$-PIV data sets. We find that the bifurcation commences at around $W i_{\text {crit }} \approx 1$ and increases rapidly up to full asymmetry at around $W i=1.8$. In contrast, the bifurcation in the work of Pathak and Hudson commenced at $W i_{\text {crit }} \approx 2$, and developed very gradually with increasing $W i$. The reason for this difference is likely to originate from differences in the flow geometry. The cross-slot of Pathak and Hudson had a larger characteristic dimension $(w=1 \mathrm{~mm})$ compared with ours $(w=0.2 \mathrm{~mm})$. The larger inertial contributions to the flow in their case may have suppressed the onset of the bifurcation..$^{28,44} 3 \mathrm{D}$ effects may also play a role; the geometry of Pathak and Hudson was significantly shallower (aspect ratio $\alpha=d / w=0.53)$ than ours $(\alpha=5.25)$.

In our work the onset of the flow bifurcation at $W i_{\text {crit }} \approx 1$ corresponds to a shear rate in the channel of $\dot{\gamma}_{c r i t} \approx 0.8 \mathrm{~s}^{-1}$, which is soon after the onset of the stress plateau in the flow curve and close 
to the development of the shear banded flow profile across the inlet/ outlet channels of the cross-slot, see Fig. 8a. However, we should be clear that we do not attribute the bifurcation to the onset of shear banding, since similar-looking phenomena have been reported in non-shear banding fluids..$^{23,42,44,45}$ Rather we suggest that shear banding in the $\mathrm{CPyCl}$ solution (specifically the shape of the flow curve shown in Fig. 3b) results in the bifurcation that we observe assuming its unusual functional form. Shear localization at the sharp re-entrant corners of the cross-slot device is likely to make an important contribution to this, which will be discussed and become clear in the following sections. Reports of bifurcations in cross-slot flow with constant-viscosity polymer solutions have shown a square-root dependency on the flow strength, consistent with classical bifurcation theory for viscous fluids. ${ }^{42,44,45}$ However, our measurements follow a different trend. Pathak and Hudson ${ }^{23}$ found a square-root dependency for the cross-slot flow bifurcation of a non-shear banding viscoelastic $\mathrm{CTAB} / \mathrm{NaSal}$ solution, ${ }^{55}$ however they also note that the corresponding bifurcation in the shear banding $\mathrm{CPyCl} / \mathrm{NaSal}$ solution follows a different scaling for reasons that they do not try to explain. Recent microfluidic measurements in rectilinear shearing flows certainly show that the stress-plateau and shear banding behaviour is much more pronounced in the $\mathrm{CPyCl} / \mathrm{NaSal}$ fluid than in $\mathrm{CTAB} / \mathrm{NaSal} .{ }^{55} \mathrm{We}$ speculate that the different scaling with $\left(W i-W i_{\text {crit }}\right)$ arises from an interplay between extensional strain-hardening in the fluid near the stagnation point and shear banding/localization in the upstream and downstream microchannels and around the re-entrant corners. The onset of shear banding and the very small increase in the total system stress associated with changes in the flow rate enhance the subsequent development of the asymmetry with increasing $\mathrm{Wi}$. Detailed investigation of this change in the scaling with $(\mathrm{Wi}-$ $W i_{\text {crit }}$ ) must await full three-dimensional numerical simulations with microstructural models that describe the viscoelaticity and shear banding features of wormlike micellar solutions.

Transition to unsteady flow. The regime of completely asymmetric flow $(D Q=1)$ spans at least two decades of volumetric flow rate, as shown by the $\mu$-PIV vector flow field maps presented in Fig. 10, for flow rates up to $Q=200 \mu \mathrm{L} \mathrm{min} \min ^{-1}$ (Re $\approx$ $0.025, W i=200)$. Interestingly, in this asymmetric flow regime we observe the development of stagnant regions, similar to recirculating lip vortices, near the re-entrant upstream corners of the cross-slot geometry (note, for example, the top-right and bottom-left walls of the inlet channels in Fig. 10a-c). Such viscoelastic secondary flows upstream of a $90^{\circ}$ corner were first reported by Chono and Iemoto ${ }^{84}$ for the flow of a $1 \%$ aqueous polyacrylamide solution in a macroscale ' $L$ '-bend, and were later modelled by the same authors using a White-Metzner constitutive equation. ${ }^{61,85}$ More recently, Gulati et al. reported similar lip vortices in the flow of shear-thinning viscoelastic semi-dilute poly(ethylene oxide) and DNA solutions around abrupt $90^{\circ}$ bends in microchannels. ${ }^{\mathbf{8 6}, 87}$ However, we are unaware of any previous reports of such elastic secondary flow phenomena in micellar fluids. We have briefly investigated the growth of the lip vortices with Weissenberg number for our wormlike micellar solution, and we present the results as Supplementary Information, see Fig. SI1 and also Movie SI4. $\dagger$ Gulati et al. argue that the lip vortex is generated due to the curvature of the streamlines around the $90^{\circ}$ bend and the elastic
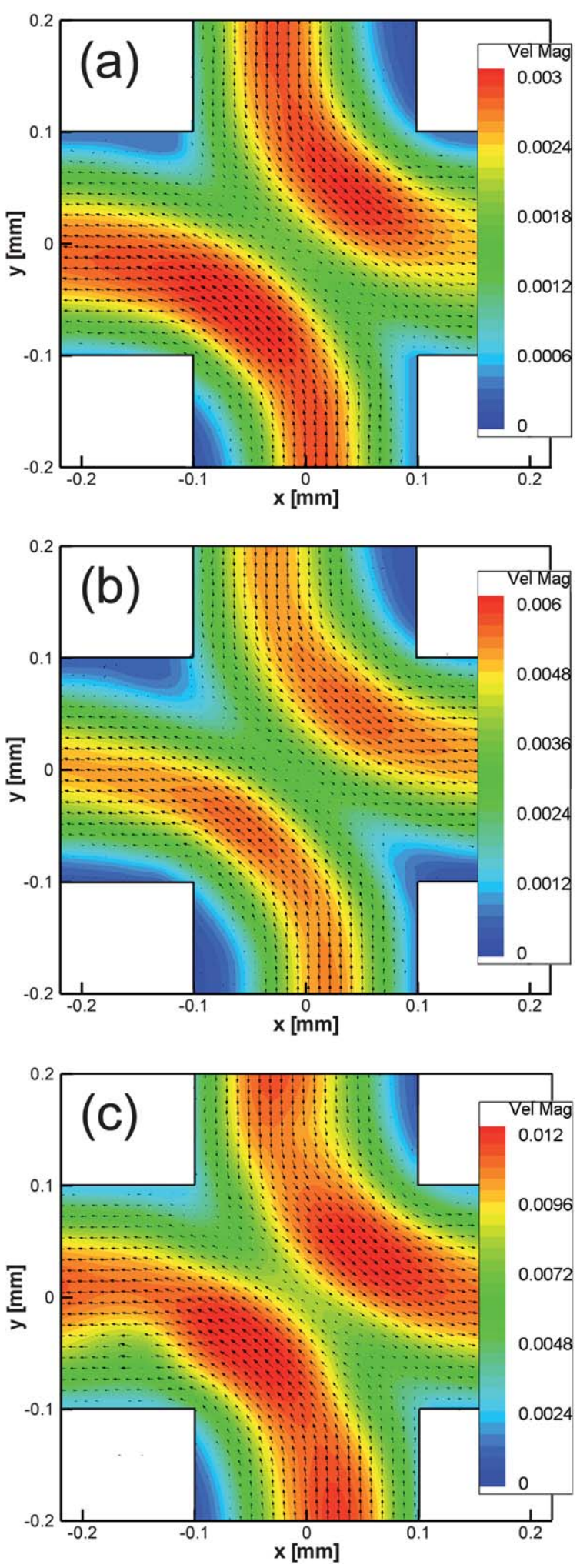

Fig. 10 Fully asymmetric flow in the cross-slot geometry for $100 \mathrm{mM}$ CPyCl/60 mM NaSal: (a) $Q=50 \mu \mathrm{L} \mathrm{min}^{-1}, R e \approx 0.003$, (b) $Q=100 \mu \mathrm{L}$ $\min ^{-1}, R e \approx 0.01$, (c) $Q=200 \mu \mathrm{L} \mathrm{m^{-1 }}, R e \approx 0.04$. Colour scale bars represent the velocity magnitude and are in $\mathrm{ms}^{-1}$.

normal stress difference that generates a tension along the streamline, but that shear-thinning is also required to reduce the stress gradients near the corner. ${ }^{87}$ 

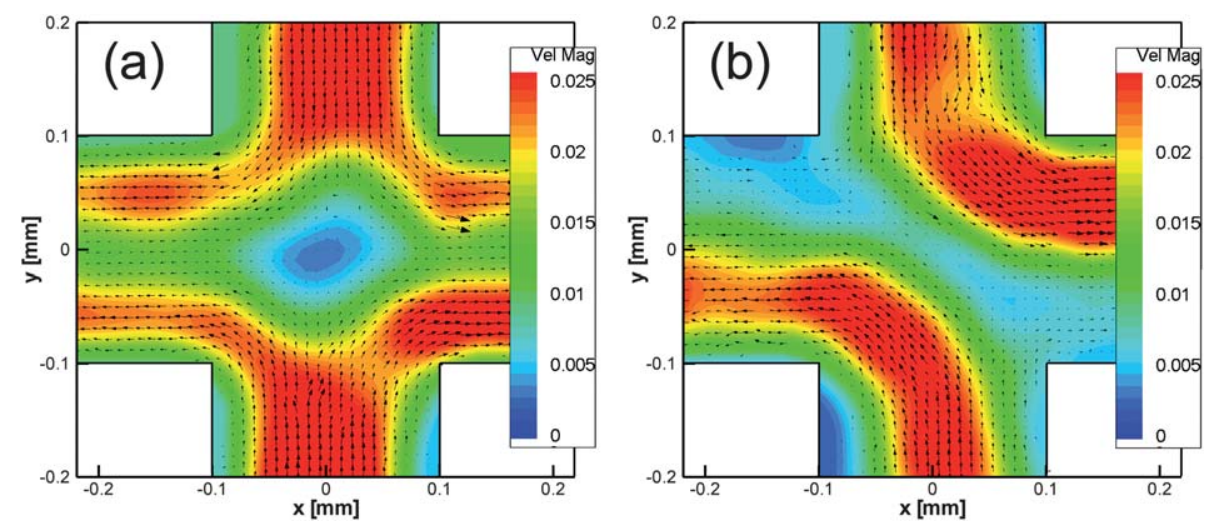

Fig. 11 Representative examples of the velocity field in the cross-slot in the time-dependent flow regime at $Q=500 \mu \mathrm{L} \mathrm{min}^{-1}, R e \approx 0.15, W i=500$ at two different times (a) and (b). Colour scale bars represent the velocity magnitude and are in $\mathrm{ms}^{-1}$.

In the asymmetric flow regime the flow remains steady; once the bifurcation has become established to either side of the cross-slot, the direction of the bifurcation generally does not change, and remains stable to imposed changes in the flow rate within this regime. As in the related, but geometrically asymmetric, microfluidic device of Groisman et al. ${ }^{47}$ the direction of the bifurcation only flips if a sufficiently large external perturbation is applied. However, if the flow rate is stepped down, such that the Weissenberg number becomes $W i<1$, the symmetric flow state is recovered.

If the flow rate is stepped up to $Q>350 \mu \mathrm{L} \mathrm{min}{ }^{-1}(R e \approx 0.07$, $W i=350)$ a new elastic instability arises and the flow becomes dramatically unsteady. Fig. 11 shows two examples of $\mu$-PIV vector flow field maps captured within the unsteady flow regime at a flow rate of $Q=500 \mu \mathrm{L} \mathrm{min} \min ^{-1}(R e \approx 0.015, W i=500)$. Streakline imaging of the flow in this unsteady regime is provided by Movie SI5 in the ESI. $\uparrow$ For brief moments the flow field regains some semblance of symmetry; in Fig. 11a the inlet flow splits almost evenly into the two opposing outlet channels and we see a stagnation point located near the centre of the cross. However, in contrast to the flow field observed in the steady symmetric flow regime for $\mathrm{Wi}<1$ (Fig. 7a), here we observe a clear region of low velocity fluid exiting along the middle of the outflow channels, with bands of high flow velocity on either side of the central band. This kind of non-monotonic variation in the velocity profile is very similar to that resulting from the flow modification associated with the strong stretching of macromolecules in semi-dilute polymer solutions, ${ }^{50}$ and likely indicates the near complete orientation and alignment of micelles that pass close to the stagnation point. In polymer solutions, where such modified flow fields have been observed under steady conditions, the alignment of molecules along the stagnation point streamline (confirmed by flow-induced birefringence measurements) is accompanied by a large increase in the extensional viscosity of the fluid. ${ }^{50,52}$ The modification to the kinematics results from the very high viscosity of stretched fluid elements located along the outflow axis acting to retard the flow of neighbouring planes of fluid, and effectively dividing the exit channels in two. Hence, we observe what appears to be two adjacent Poiseuille-like flow profiles across the channel.

In this rapidly time-varying flow regime, the pseudosymmetric flow field illustrated by Fig. 11a is short lived and within a maximum of one or two seconds becomes replaced by a bifurcated flow field (e.g. Fig. 11b), which can flip from the left to the right-hand side, briefly passing through the symmetric state, occasionally maintaining symmetry for a short period of time (typically $<1 \mathrm{~s}$ ). Visually, there appears to be no unique characteristic time scale for these fluctuations; a pseudo-steady flow could be observed for periods of several seconds $\left(\sim \lambda_{M}\right)$, only to be replaced by periods of rapid fluctuations. In Section 3.2.3 we quantify the temporal structure of the instability using timeresolved pressure drop measurements.

Finally, we note that the onset of the time-dependent flow regime in the cross-slot geometry occurs at a shear rate of $\dot{\gamma} \approx$ $280 \mathrm{~s}^{-1}$, which is very close to the end of the stress plateau observed in the steady flow curve. This provides further corroborating evidence that the region of steady bifurcated flow is influenced by shear banding in the microchannels.

\subsubsection{Flow-induced birefringence observations and measure-} ments. To investigate the role of microstructural orientation of micelles in the cross-slot instability mechanism, we also made full-field measurements of the flow induced birefringence in the micellar solution using an ABRIO imaging system, ${ }^{55,71}$ see Section 2.6. In this Section we report the results obtained within the three flow regimes described previously.

Symmetric flow. Fig. 12 presents the results of full-field birefringence observations made on the $100 \mathrm{mM} \mathrm{CPyCl} / 60 \mathrm{mM}$ $\mathrm{NaSal}$ solution during the regime of stable symmetric flow ( $\mathrm{Wi}<$ 1). The colour scale represents the retardation, $R$ expressed in $\mathrm{nm}$, through the micellar solution, which can be converted to birefringence $\Delta n$ by dividing by the depth, $d$, of the cross-slot flow cell:

$$
R=d \Delta n
$$

Eqn (16) assumes the flow is homogenous through the depth of the channel in the direction of light propagation, with negligible effects from the inside surfaces of the glass end walls. This is likely to be reasonable, since we have shown that the flow becomes plug-like in the orthogonal narrow direction across the channel and we assume that a similar profile develops through the channel depth. 

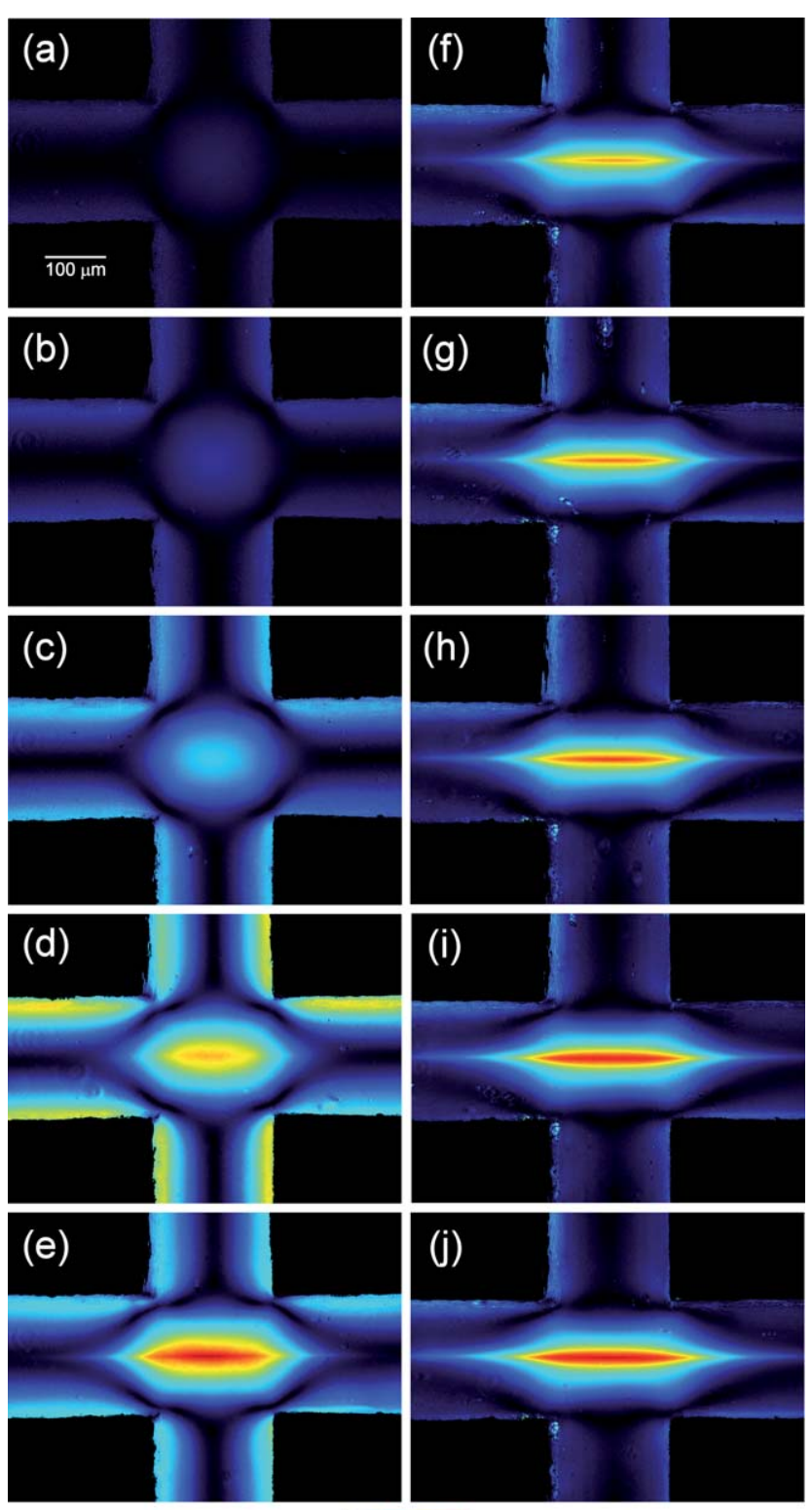

1

Fig. 12 Flow induced optical retardation, $R(x, y)$ for $100 \mathrm{mM} \mathrm{CPyCl} / 60$

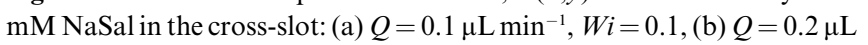
$\min ^{-1}, W i=0.2$, (c) $Q=0.3 \mu \mathrm{L} \mathrm{min}^{-1}, W i=0.3$, (d) $Q=0.4 \mu \mathrm{L} \mathrm{min}^{-1}, W i=$ 0.4 , (e) $Q=0.5 \mu \mathrm{L} \mathrm{min}^{-1}, W i=0.5$, (f) $Q=0.6 \mu \mathrm{L} \mathrm{min}^{-1}, W i=0.6$, (g) $Q=$ $0.7 \mu \mathrm{L} \mathrm{min}-1, W i=0.7$, (h) $Q=0.8 \mu \mathrm{L} \mathrm{min}-1, W i=0.8$, (i) $Q=0.9 \mu \mathrm{L}$ $\min ^{-1}, W i=0.9$, (j) $Q=1.0 \mu \mathrm{L} \mathrm{min}-1, W i=1.0$. The Reynolds number in all Figures is $R e<10^{-5}$. The colour scale represents optical retardation in the range $0-10 \mathrm{~nm}$ in Fig. (a)-(e) and 0-20 nm in Fig. (f) to (j).

At very low flow rates $\left(Q \leq 0.3 \mu \mathrm{L} \mathrm{min}^{-1}, W i \leq 0.3\right.$, Fig. 12a-c) the birefringence increases in a diffuse, approximately circular, region surrounding the stagnation point. The intensity of the birefringence near the stagnation point is comparable to that induced by shear along the channel walls, indicating that molecular deformation of micelles is relatively low and both shearing and extensional kinematics are of equal importance in the cross-slot. When the flow rate is increased to $Q=0.4 \mu \mathrm{L}$ $\min ^{-1}(W i=0.4$, Fig. 12d) a localised region of more intense birefringence starts to develop near to the stagnation point, extending towards the exit channels of the cross-slot. As the flow rate is increased further, beyond $W i \approx 0.5$ (Fig. $12 \mathrm{e}-\mathrm{j}$ ), the birefringent strand centred on the stagnation point increases significantly in length and intensity. Now the stagnation point birefringence clearly dominates over the shear-induced birefringence along the channel walls, indicating that the wormlike micelles are becoming significantly more oriented by the extensional flow field at the stagnation point.

Line profiles taken through the stagnation point along the inflow direction ( $x=0$ axis) show the power of the stagnation point to induce strong micellar alignment by the development of a cusp in the birefringence for $W i>0.5$ (Fig.13a), as also reported by Pathak and Hudson. ${ }^{23}$ Profiles taken through the birefringent strands along the outflow direction ( $y=0$ axis) for $W i>0.5$ are shown in Fig. 13b. With increasing $W i$, the birefringence becomes almost constant between the channel entrances $(-0.1 \mathrm{~mm} \leq x \leq 0.1 \mathrm{~mm}$, marked by dashed black lines). However, within the exit channels the birefringence decays exponentially, as shown by the fitted solid black lines. The characteristic decay lengths of the fitted curves can be divided by the known centre-line flow velocity (from $\mu$-PIV measurements) to obtain a measure of the decay time of the birefringence (in the absence of imposed shearing or extensional velocity gradients) as material elements are convected along the centreline of the exit channels. Averaging the values obtained from the four curves in Fig.13b, we find a mean life time for the birefringence of $\tau=2.6$ $\pm 0.2 \mathrm{~s}$, close to the values of $\lambda_{M}$ and $\lambda_{C a B E R}$, as expected.

The magnitude of the birefringence (measured at the stagnation point) increases in a sigmoidal fashion with $W i$ (and $\dot{\varepsilon}_{n o m}$ ) in the symmetric flow regime, as shown in Fig.13c. The birefringence plateaus at a value of $\Delta n \approx-2 \times 10^{-5}$ at $W i \approx 1.2$. While this value agrees well with that reported by Pathak and Hudson at equal $W i$, the birefringence that they measured increased to a significantly higher plateau value of $\Delta n \approx-6 \times 10^{-5}$ at $W i \approx 3$. In our experiment, the bifurcation to a steady asymmetric flow commences before such a high Weissenberg number can be attained. Fig. $13 \mathrm{c}$ also shows the stress at the stagnation point as a function of $W i$ and $\dot{\varepsilon}_{\text {nom }}$, determined using the SOR (eqn (4)). In Fig. 13d, this data has been used to compute an apparent extensional viscosity $\left(\eta_{E, a p p}\right)$ and is shown alongside the shear viscosity $(\eta(\dot{\gamma}))$ obtained from the AR-G2 cone-and-plate rheometer. The inset in Fig. 13d shows that the Trouton ratio $\left(T r=\eta_{E, a p p} / \eta\right)$, which increases monotonically with $W i$ up to a value of $T r \approx 30$, is of similar order to the value obtained by CaBER (see Fig. $4 \mathrm{~b}$ ).

Steady bifurcated flow. Fig. 14 shows the evolution of the birefringent strand as the flow rate is increased into the steady bifurcated flow regime. Paralleling the kinematic measurements made using $\mu$-PIV, as the flow rate is increased the orientation of the birefringent strand rotates by ever-increasing degrees (Fig. 14a-i). Eventually, the completely asymmetric state $(D Q=1)$ is achieved at a flow rate of $Q=2 \mu \mathrm{L} \mathrm{min}{ }^{-1}$ ( $W i=2$, Fig. 14j). The onset of the fully asymmetric state is marked by a narrow and weaker birefringent strand located between diagonally opposed corners of the cross-slot and a significant drop in the magnitude of the birefringence, due to the complete loss of the elongational flow near the stagnation point. The birefringence in Fig. $14 \mathrm{j}$ is induced solely by the shear stress generated along the dividing streamline as fluid 

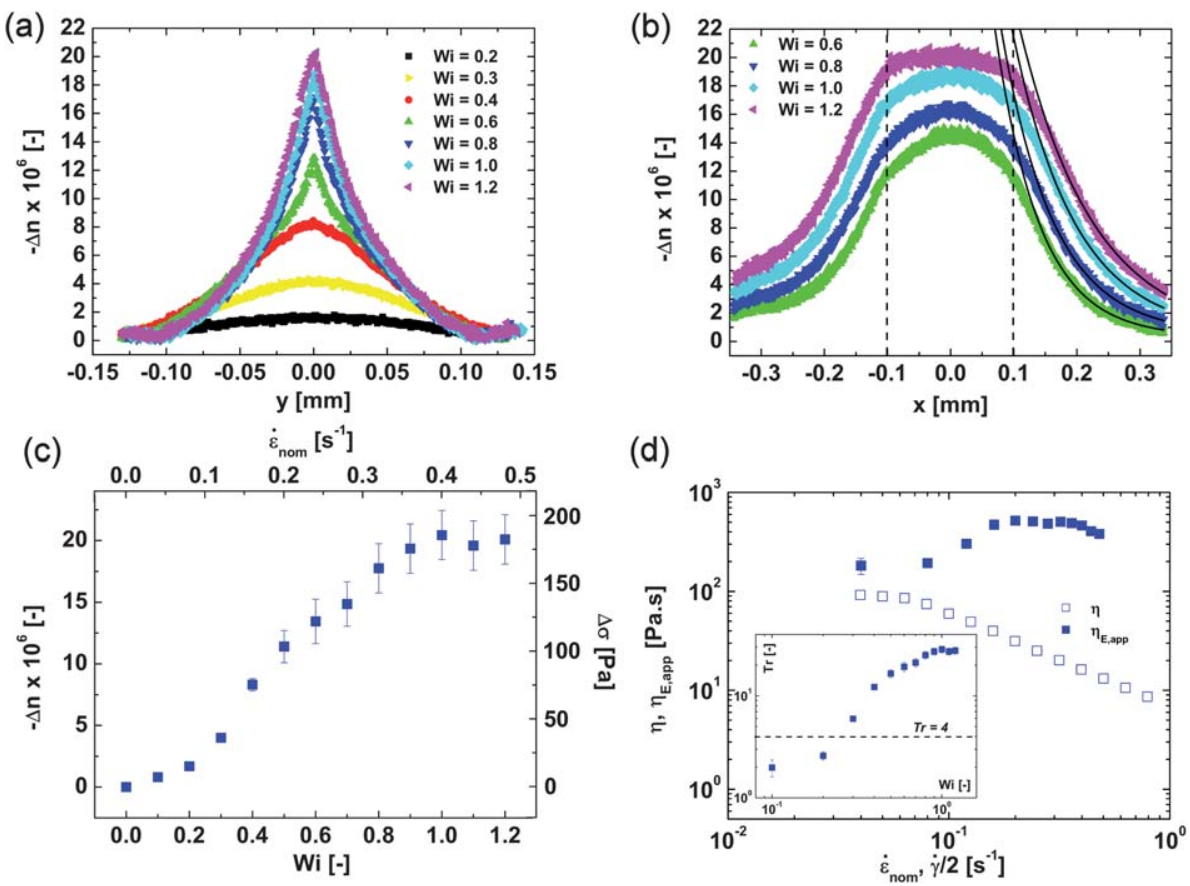

Fig. 13 (a) Profiles across birefringent bands along the inflow axis $(x=0 \mathrm{~mm})$ for various flow rates in the symmetric flow regime. (b) Profiles along the lengths of the birefringent bands $(y=0 \mathrm{~mm}$ ). Along the outflow axis, within the exit channels (marked by dashed lines), the birefringence decays exponentially, as shown by the fitted solid lines. (c) Measured peak birefringence $(\Delta n)$ and principal stress difference $(\Delta \sigma)$ as a function of the nominal strain rate, $\dot{\varepsilon}_{\text {nom }}$, and Weissenberg number, $W i$. (d) Shear viscosity and apparent extensional viscosity as a function of $\dot{\varepsilon}_{\text {nom }}$ and, inset, the dimensionless Trouton ratio as a function of $W i$; the Trouton ratio of 4 marks that expected for a Newtonian fluid in a planar extensional flow.

flowing around opposing corners of the cross is forced to squeeze through the narrower flow cross-section.

Further increasing the flow rate within the steady fully-bifurcated flow regime increases the shear rate and shear stress on the diagonal birefringent strand and at the channel walls, resulting in an increased birefringence signal, as shown in Fig. 15a-c. At $Q=20 \mu \mathrm{L} \min ^{-1}$ (Fig. 15c) we begin to observe a manifestation of the lip vortex observed in the $\mu$-PIV measurements made within this flow regime (see Fig.10 and Supporting Information). Note how the birefringence along the top-left and bottom-right inlet channel walls detaches prior to the re-entrant corner. As the flow rate is increased further (Fig. 15d-f) the re-entrant lip vortex grows upstream to such an extent that the attachment point extends beyond the field of view. Also, the diagonal birefringent strand is superseded by a progressive transition to a state in which the fluid is highly birefringent and aligned across a significant portion of both the inlet and outlet channels, e.g. Fig. $15 \mathrm{e}-\mathrm{f}$. This transition occurs at channel shear rates of $\dot{\gamma} \approx 80 \mathrm{~s}^{-1}$, closely corresponding to the start of the upturn in the steady flow curve, i.e. close to the end of the stress plateau (see Fig. 3b). It appears that this state may be the channel-flow equivalent to the formation of a single high-shear-rate band as observed in Couette and cone-and-plate flows. At this point the micellar fluid behaves as a flow-aligned viscoelastic polymer solution with streamlines that curve around the re-entrant corners of the geometry. It has recently been demonstrated in Taylor-Couette flow of a shear banding micellar solution that such conditions of high micelle alignment and streamline curvature can give rise to time-dependent purely elastic instabilities. ${ }^{88}$ Note that at these high flow rates $\left(Q \geq 100 \mu \mathrm{L} \mathrm{min}{ }^{-1}\right)$ the flow in the cross-slot starts to become slightly erratic, which accounts for the slightly streaky appearance of the images.

Unsteady, time-dependent flow. Fig. 16 shows a series of birefringence images captured sequentially at $\sim 5 \mathrm{~s}$ intervals with a fixed volume flow rate of $Q=350 \mu \mathrm{L} \min ^{-1}(R e \approx 0.07$, $W i=350)$. In this unsteady flow regime, quantitative birefringence measurements are difficult to obtain because of the spatiotemporal fluctuations in the magnitude of the flow birefringence and the time-averaging associated with the need to analyze five consecutive frames. ${ }^{55,71}$ Instead we present individual grey-scale images of the retardation, which can be captured more rapidly with less time-averaging, and clearly show the time-dependent nature of the flow. In all of the images we observe that the inlet and outlet channels are essentially filled with birefringent fluid, however close to each inlet channel entrance is a dark, roughly triangular "void", where the birefringence is low. The two voids are separated by the central birefringent region. The voids oscillate from side to side with the changes in direction of the bifurcation (compare e.g. Fig. 16d and Fig. 16f). Occasionally a near symmetric configuration is briefly established, e.g. Fig. 16e, Fig. 16i. These observations closely mirror the $\mu$-PIV measurements presented in Fig.11, except that the local void regions in the birefringence (corresponding to regions of very low molecular deformation) can not be observed in the kinematic data.

3.2.3. Pressure drop and extensional viscometry. In addition to local measurements of the kinematics and optical anisotropy, we also made measurements of the integrated pressure drop 

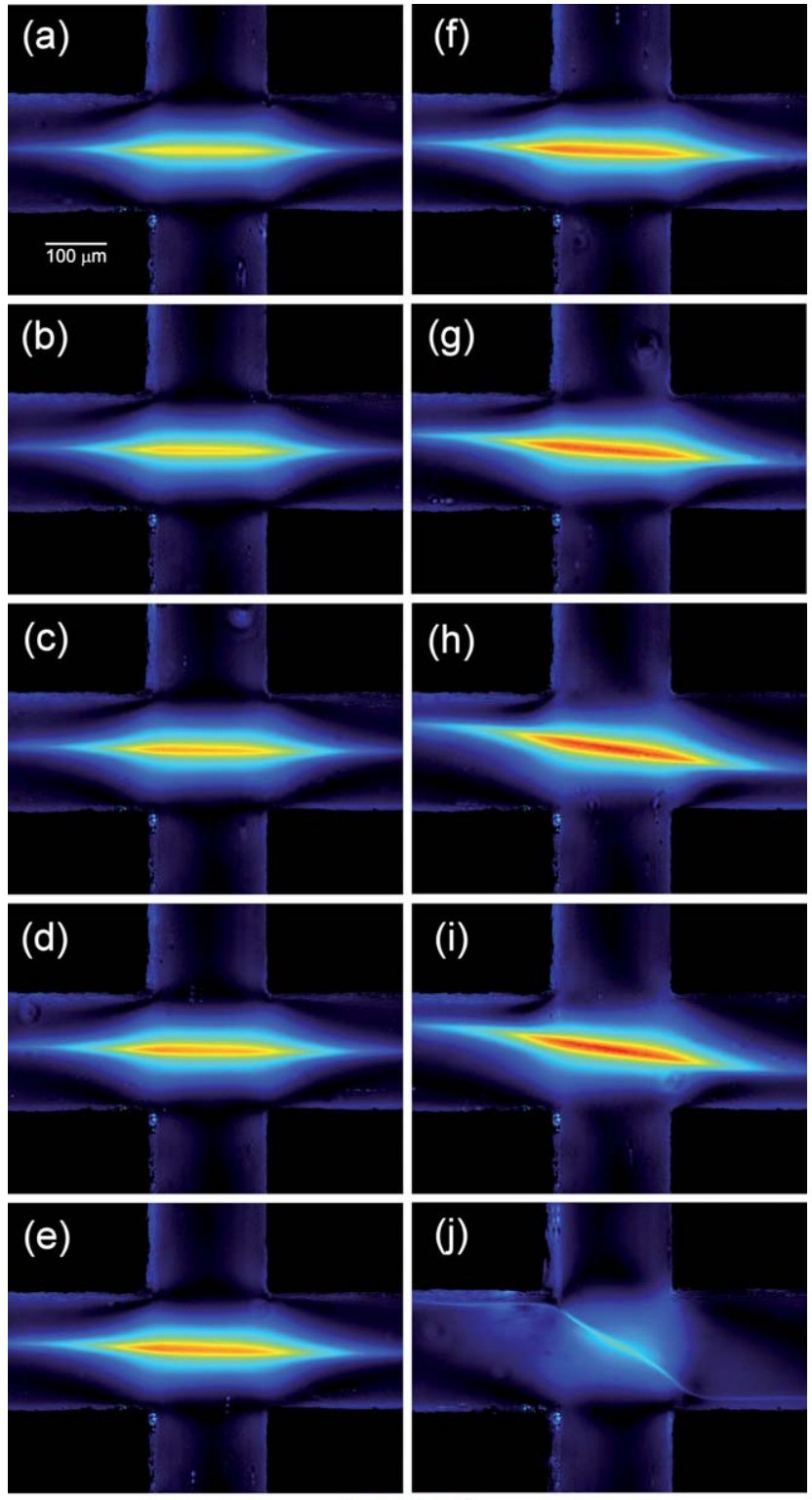

1.1

Fig. 14 Flow induced optical retardation for $100 \mathrm{mM} \mathrm{CPyCl} / 60 \mathrm{mM}$ $\mathrm{NaSal}$ in the cross-slot following the onset of steady asymmetric flow: (a) $Q=1.1 \mu \mathrm{L} \mathrm{min}{ }^{-1}, W i=1.1$, (b) $Q=1.2 \mu \mathrm{L} \mathrm{min}-1, W i=1.2$, (c) $Q=1.3$ $\mu \mathrm{L} \mathrm{min}{ }^{-1}, W i=1.3$, (d) $Q=1.4 \mu \mathrm{L} \mathrm{min}^{-1}, W i=1.4$, (e) $Q=1.5 \mu \mathrm{L} \mathrm{min}^{-1}$, $W i=1.5$, (f) $Q=1.6 \mu \mathrm{L} \mathrm{min}^{-1}, W i=1.6$, (g) $Q=1.7 \mu \mathrm{L} \mathrm{min}^{-1}, W i=1.7$, (h) $Q=1.8 \mu \mathrm{L} \mathrm{min}^{-1}, W i=1.8$, (i) $Q=1.9 \mu \mathrm{L} \mathrm{min}^{-1}, W i=1.9$, (j) $Q=2.0$ $\mu \mathrm{L} \mathrm{min}{ }^{-1}, W i=2.0$. The Reynolds number in all Figures is $R e<10^{-5}$. The colour scale represents optical retardation in the range $0-30 \mathrm{~nm}$ in all images.

associated with the viscoelastic flow through the cross-slot geometry. The pressure drop measured as a function of flow rate is shown for a typical experiment with the $\mathrm{CPyCl} / \mathrm{NaSal}$ solution in Fig.17a. Under conditions of symmetric flow, there is a measurable excess pressure drop $\left(\Delta P_{\text {excess }}\right)$ associated with the extensional component of the flow, i.e. $\Delta P_{\text {total }}>\Delta P_{\text {shear }}$. However, this excess pressure drop reduces markedly as the flow rate increases into the steady bifurcated flow regime. At flow rates beyond which the bifurcation is complete, i.e. $D Q \approx 1$,
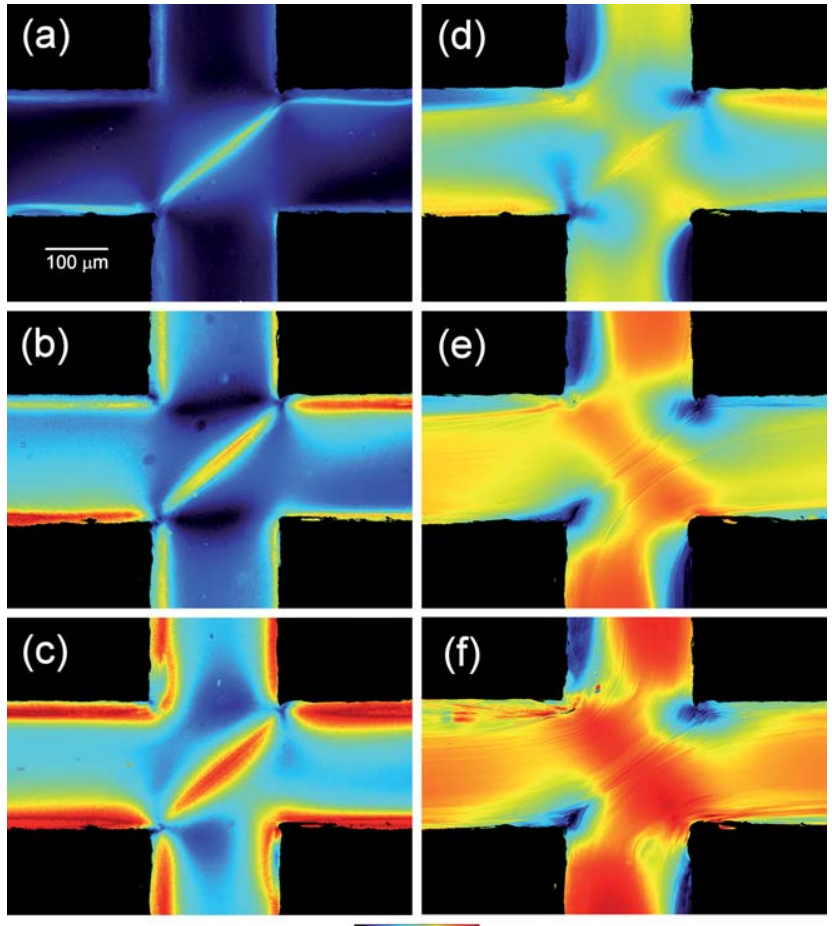

1.1

Fig. 15 Flow induced retardation for $100 \mathrm{mM} \mathrm{CPyCl} / 60 \mathrm{mM}$ $\mathrm{NaSal}$ in the cross-slot following the onset of time-dependent 3D flow: (a) $Q=5 \mu \mathrm{L} \mathrm{min}{ }^{-1}, R e \approx 2 \times 10^{-5}$, (b) $Q=10 \mu \mathrm{L} \mathrm{min}{ }^{-1}, R e \approx 7 \times 10^{-5}$, (c) $Q=20 \mu \mathrm{L} \mathrm{min}{ }^{-1}, R e \approx 3 \times 10^{-4}$, (d) $Q=50 \mu \mathrm{L} \mathrm{min}^{-1}, R e \approx 0.002$, (e) $Q=100 \mu \mathrm{L} \mathrm{min}{ }^{-1}, R e \approx 0.006$, (f) $Q=200 \mu \mathrm{L} \mathrm{min}{ }^{-1}, R e \approx 0.025$. The colour scale represents optical retardation of $0-50 \mathrm{~nm}$ in Fig. (a)-(c) and $0-80 \mathrm{~nm}$ in Fig. (d)-(f).

there is a significant range of $\dot{\varepsilon}_{\text {nom }}$ over which $\Delta P_{\text {excess }}$ is essentially zero. This is consistent with expectations; when $D Q \approx 1$ the total pressure drop $\left(\Delta P_{\text {total }}\right)$ across the cross-slot also corresponds to flow around a single $90^{\circ}$ corner of the cross, equivalent to $\Delta P_{\text {shear }}$. As the flow rate increases further and we approach the time-dependent region, once again we begin to measure a nonzero excess pressure associated with the strongly flow-aligned viscoelastic state.

In Fig. $17 \mathrm{~b}$ we use the excess pressure drop within the symmetric flow regime to compute an apparent extensional viscosity $\left(\eta_{E, \text { app }}\right)$ according to eqn (10). The data presented is the average of three separate measurements and the error bars represent the standard deviation. Also shown on Fig. $17 \mathrm{~b}$ is the shear viscosity, $\eta(\dot{\gamma})$, obtained from the AR-G2 cone-and-plate rheometer. The resulting Trouton ratio measured as a function of Weissenberg number is shown in Fig. 17c. The magnitude of the Trouton ratio that we measure from the pressure drop data in the symmetric flow regime is fairly modest, as also reported by previous authors for stagnation point flows of similar fluids. ${ }^{23,33}$ This is in contrast to filament stretching and capillary breakup (CaBER) experiments, in which Trouton ratios $T r \sim O(100)$ have been reported. ${ }^{63,89}$ Indeed earlier we measured a Trouton ratio of up to approximately 40 for our own test fluid using the CaBER device (Fig. 4b) and up to around 30 using the stress-optical rule (SOR) and measurements of birefringence in the cross-slot. The form of the curve in Fig. 17c is also strange; broadly we observe 

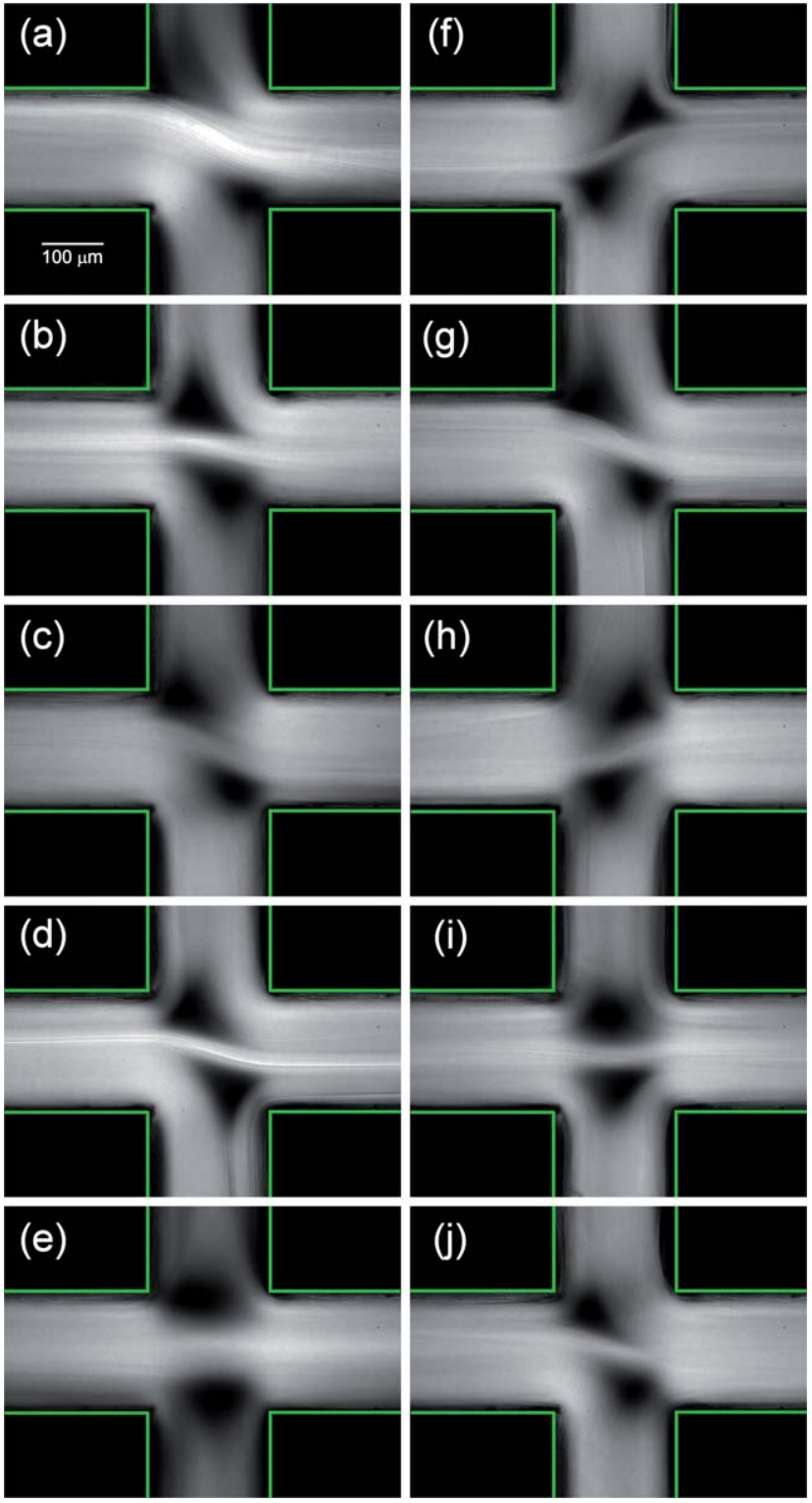

Fig. 16 (a)-(j) Flow induced retardation imaged through crossed polarizers for $100 \mathrm{mM} \mathrm{CPyCl} / 60 \mathrm{mM} \mathrm{NaSal}$ in the cross-slot in the timedependent flow regime at $Q=350 \mu \mathrm{L} \mathrm{min} \min ^{-1}(R e \approx 0.07, W i=350$ ). Successive images were captured at intervals of $\sim 5 \mathrm{~s}$. The cross-slot walls have been drawn in for clarity.

an initial increase in $T r$ at low $W i$ followed by a gradual reduction in $\operatorname{Tr}$ for $W i>0.4$. These differences between $\eta_{E, a p p}$ determined from the birefringence data and the pressure drop data are most likely due to the fact that the pressure drop is a bulk, integrated measurement that reflects the contributions of all the material elements flowing through the cross-slot. On the other hand, the birefringence measurement allows a direct local evaluation of the extensional stress difference near the stagnation point using the SOR (eqn (4)). In such a strongly shear-thinning and shear banding fluid it is likely that the shear contributions to the pressure drop will vary greatly depending on the flow configuration, i.e. between the two bounding values of $\Delta P_{\text {shear }}$ and $\Delta P_{\text {total }}$ evaluated in eqn (10). Deconvoluting the shear and extensional contributions using eqn (10) may thus be ill-posed,
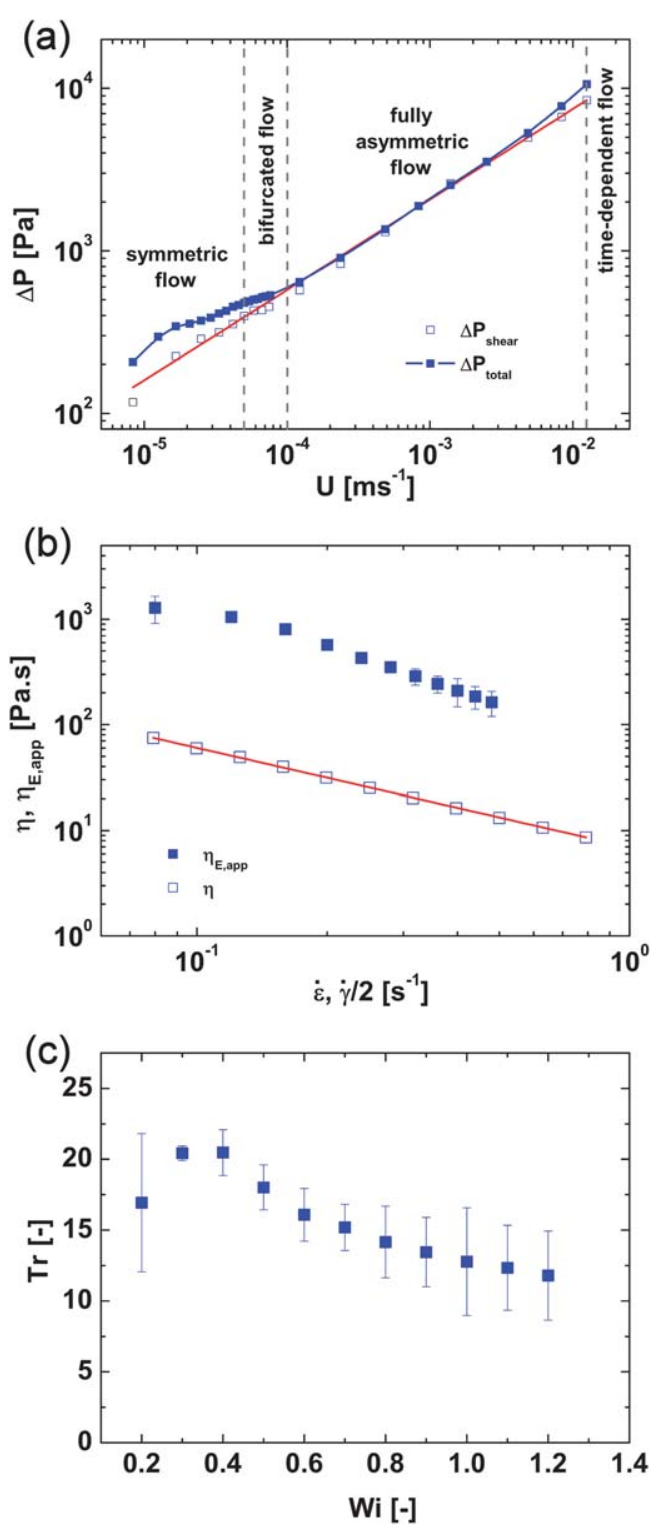

Fig. 17 (a) Typical evolution of the pressure drop measured across the cross-slot as a function of the superficial flow velocity for $100 \mathrm{mM} \mathrm{CPyCl} /$ $60 \mathrm{mM} \mathrm{NaSal}$. The solid red line is a power-law fit to $\Delta P_{\text {shear }}$ with an exponent of $n \approx 0.56$ (b) Steady shear and apparent extensional viscosity as a function of strain rate; the shear viscosity was determined using coneand-plate rheometry and is fitted with a power-law (solid red line). (c) Trouton ratio as a function of $W i$, determined from (b).

leading to the limited utility of such bulk measurements in probing the elongational properties of these extremely shearthinning micellar fluids.

Naturally, in the bifurcated flow regime, when the excess pressure approaches zero the extensional viscosity and Trouton ratio would also become immeasurably small, which is what we observe for Newtonian fluids in our cross-slot. However, in this limit, the definition in eqn (10) is entirely meaningless and provides no description of the extensional properties of the fluid since, when the flow is fully bifurcated, there is no well-defined extensional component in the flow field. Similar behaviour was reported for a variety of wormlike micelle-forming surfactant 
solutions in opposed jet rheometers. ${ }^{31-33}$ Walker et al. ${ }^{33}$ explained this reduction in $\operatorname{Tr}$ as being due to either breaking or slipping of aligned micelles or to a loss of the stagnation point between the jets. However, they were not able to perform local visualization of the flow kinematics to confirm this. It appears clear from our experiment that the reduction in the excess pressure drop and hence in $\eta_{E, a p p}$ (and $T r$ ) is chiefly due to the flow bifurcation and eventual loss of the stagnation point when complete flow asymmetry is realized. We note also that the birefringence essentially saturates in the bifurcated regime and drops significantly when the flow becomes fully asymmetric (Fig. 14), which is consistent with a reduction in the micellar strain and elongational stress. The reduction in both $\Delta P_{\text {excess }}$ and $\Delta n$ within the steady flow bifurcation regime indicates that the driving mechanisms may be energy minimization and the resistance of micelles to orient, due to entropic considerations. It appears that by bifurcating at $W i>1$, a given volume flow rate can be maintained for both lower pressure and lower micellar alignment than if the flow remained symmetric. Therefore our experimental results with wormlike micellar solutions appear to demonstrate a similar bifurcation mechanism to that proposed by Rocha et al. ${ }^{45}$ from simulations with FENE models. However, the reason for the critical $W i$ for the bifurcation is not the overcoming of an energy barrier, since also in agreement with their work, ${ }^{45}$ we find the symmetric flow state is recovered if $W i$ is reduced to less than the
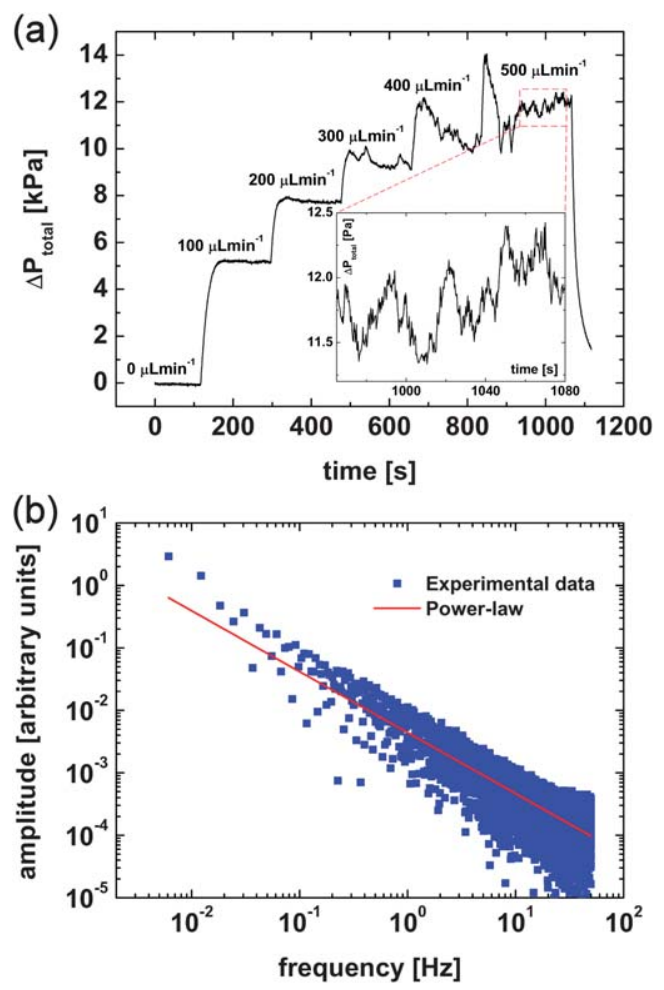

Fig. 18 (a) Pressure drop as a function of time as the flow rate is incremented through the steady bifurcated regime to the time-dependent flow regimes, resulting in rapid fluctuations in the pressure for flow rates greater than $Q=300 \mu \mathrm{L} \mathrm{min}{ }^{-1}$ (inset shows detail of fluctuation at $Q=$ $500 \mu \mathrm{L} \mathrm{min}{ }^{-1}$ ). (b) The Fourier transform of the pressure trace at $Q=$ $500 \mu \mathrm{L} \mathrm{min}{ }^{-1}$ shows a broad power law regime inversely proportional to the frequency, indicating random fluctuations. critical value ( $W i_{\text {crit }} \approx 1$, in our case), suggesting that for $W i<1$ symmetric flow is the lowest energy state. For our micellar test fluid the highly localized shear at the channel walls and corners provides a "self-lubricating" effect, hence causing the bifurcation to develop more rapidly than it would in a less shear-thinning fluid. This perhaps explains the unusual functional form of the bifurcation scaling in this system.

Fig. 18a shows the total pressure drop measured as a function of time as the flow rate is increased incrementally from the steady bifurcated to the time-dependent flow regime. In the steady bifurcated regime $\left(Q<300 \mu \mathrm{L} \mathrm{min}{ }^{-1}\right)$ the pressure drop quickly reaches a smooth plateau value after a flow rate is imposed. However, at flow rates in the time-dependent flow regime $(Q>$ $300 \mu \mathrm{L} \mathrm{min}{ }^{-1}$ ) the total pressure drop fluctuates erratically, reflecting the unsteady nature of the flow. The Fourier transform of the enlarged portion of the pressure drop measured at $Q=$ $500 \mu \mathrm{L} \mathrm{min}{ }^{-1}$ (Fig. 18a inset) is inversely proportional to frequency (Fig. 18b), indicating that pressure fluctuations are aperiodic. Aperiodic time-dependent flow of wormlike micellar solutions has been reported previously ${ }^{23,90}$ and hints that the flow in this regime may be chaotic, although the sensitivity to initial conditions required to confirm this is hard to demonstrate for flow in the cross-slot.

\section{Conclusions}

We have used a microfluidic cross-slot device to test the response of a well-characterized model entangled wormlike micellar solution to a well-defined stagnation point extensional flow field. We find that for low flow rates such that $W i<1$, the flow field remains symmetric, and an excess pressure drop can be measured from which a bulk measure of the apparent extensional viscosity can be estimated. This low $W i$ symmetric flow regime is accompanied by the growth of a slender birefringent strand centred along the stagnation point streamline of the exit channel. Measurements of the birefringence near the stagnation point allow a second, local, estimate of the apparent extensional viscosity to be obtained using the stress-optical rule. As the Weissenberg number is increased beyond $W i \approx 1$, the flow field bifurcates and becomes asymmetric, an effect which is clearly demonstrated by the rotation of the birefringent strand (either clockwise or anticlockwise) about the stagnation point. This bifurcation is purely elastic in origin since the Reynolds number at its onset is vanishingly small $\left(R e \approx 10^{-6}\right)$. Our results suggest the flow bifurcation is driven by energy minimization considerations; for $W i>1$ the bifurcation allows an imposed volume flow rate to be maintained for a reduced excess pressure compared with a steady symmetric extensional flow. The onset of the bifurcation closely coincides with the development of shear bands in the channels of the cross-slot and we speculate that interaction between strong strain-hardening near the stagnation point and strong shear thinning in the inlet/outlet channels and around the sharp re-entrant corners may be the cause of the unusual form of the bifurcation scaling with $\left(W i-W i_{c r i t}\right)$. Within the bifurcated flow regime, the excess pressure drop progressively reduces, eventually reaching zero at $W i \approx 2$, when the flow becomes fully asymmetric. To our knowledge such a high degree of flow asymmetry $(D Q \approx 1)$ has not been previously reported in an experimental study in the cross-slot 
geometry. Full asymmetry of the flow field is maintained over more than two decades in flow rate (closely corresponding to the range of deformation rates that define the width of the shearstress plateau in steady shear flow), up to $W i \approx 350$, after which the flow becomes time dependent. In the time-dependent flow regime, rapid fluctuations in the birefringent signal and flow field are accompanied by fluctuations in the bulk pressure drop across the cross-slot, which we find are aperiodic, suggesting the flow may be chaotic in this regime. Our observations of upstream lip vortex development in the inlet channels (the first such report for micellar solutions) suggest that a combination of flow-induced micellar alignment and streamline curvature may drive the onset of the time-dependent instability.

The rapid temporal fluctuations and mixed kinematics that result from these purely elastic instabilities have potential applications in low $R e$ mixing and as bistable switches in microfluidic devices. However, in many cases, such as lab-on-achip diagnostics or jetting applications they are often to be avoided. In the future, it may be possible to obtain the required fluid dynamics for a given application (e.g. stable flow at high rates, or a certain desired periodicity for the instability) by carefully tuning the fluid formulation (e.g. solvent viscosity, micelle length/concentration and elasticity) and flow rates. It is also well-known that streamline curvature plays an important role in the development of elastic flow instabilities. This suggests that careful design of the local geometric features of the reentrant corners may provide additional leverage for the controlled manipulation of elastic instabilities in extensional flows in these kinds of complex fluids. We note that since we have employed a canonical entangled micellar fluid with simple and well-characterized rheology, our results of kinematics, flowinduced birefringence and integrated pressure drop should serve as a benchmark data set for comparison with $2 \mathrm{D}$ and $3 \mathrm{D}$ simulations based on appropriate viscoelastic constitutive models. This will permit a significantly enhanced understanding of the mechanisms underlying both the bifurcation from steady 2D symmetric flow to steady asymmetric flow and the onset of time dependence. This is currently under investigation and is expected to be the subject of a future publication.

\section{Acknowledgements}

GHM and SJH would like to acknowledge NASA Microgravity Fluid Sciences (Code UG) for support of this research under grant NNX09AV99G. TJO gratefully acknowledges support from the NSF Graduate Research Fellowship. Funding is also acknowledged from FCT and FEDER (project PTDC/EQUFTT/71800/2006). We thank M.A. Fardin for helpful discussions and comments on the manuscript.

\section{References}

1 R. G. Larson, The Structure and Rheology of Complex Fluids, Oxford University Press, New York, 1999.

2 J.-F. Berret, in Molecular Gels: Materials with Self-Assembled Fibrillar Networks, ed. R. G. Weiss and P. Terech, Springer, Dordrecht, 2006.

3 M. E. Cates and S. J. Candau, J. Phys.: Condens. Matter, 1990, 2, 6869-6892.

4 H. Rehage and H. Hoffman, Mol. Phys., 1991, 74, 933-973.
5 J. Appell, G. Porte, A. Khatory, F. Kern and S. J. Candau, J. Phys. II, 1992, 2, 1045-1052.

6 M. E. Cates and S. M. Fielding, Adv. Phys., 2006, 55, 799-879.

7 S. Ezrahi, E. Tuval and A. Aserin, Adv. Colloid Interface Sci., 2006, 128-130, 77-102.

8 J. Yang, Curr. Opin. Colloid Interface Sci., 2002, 7, 276-281.

9 Z. Xue, C. M. Corvalan, V. Dravid and P. E. Sojka, Chem. Eng. Sci., 2008, 63, 1842-1849.

10 L. B. Smolka and A. Belmonte, J. Non-Newtonian Fluid Mech., 2003, $115,1-25$.

11 J. C. Thompson and J. P. Rothstein, J. Non-Newtonian Fluid Mech., 2007, 147, 11-22.

12 J. L. Zakin, B. Lu and H. W. Bewersdorff, Rev. Chem. Eng., 1998, 14, 253-320.

13 F.-C. Li, Y. Kawaguchi, B. Yu, J.-J. Wei and K. Hishida, Int. J. Heat Mass Transfer, 2008, 51, 835-843.

14 S. Kefi, J. Lee, T. Pope, P. Sullivan, E. Nelson, A. Hernandez, T. Olsen, M. Parlar, B. Powers, A. Wilson and A. Twynam, Oilfield Review, 2004, 10-16.

15 A. J. Muller, M. F. Torres and A. E. Saez, Langmuir, 2004, 20, 3838 3841.

16 J. T. Padding, W. J. Briels, M. R. Stukan and E. S. Boek, Soft Matter, 2009, 5, 4367-4375.

17 S. Chen and J. P. Rothstein, J. Non-Newtonian Fluid Mech., 2004, 116, 205-234.

18 N. Z. Handzy and A. Belmonte, Phys. Rev. Lett., 2004, 92, 124501.

19 M. S. Turner and M. E. Cates, J. Phys.: Condens. Matter, 1992, 4, 3719-3741.

20 M. Vasudevan, E. Buse, D. L. Lu, H. Krishna, R. Kalyanaraman, A. Q. Shen, B. Khomami and R. Sureshkumar, Nat. Mater., 2010, 9, 436-441.

21 T. Takahashi and D. Sakata, J. Rheol., 2011, 55, 225-240.

22 N. Dubash, J. Cardiel, P. Cheung and A. Q. Shen, Soft Matter, 2011, 7, 876-879.

23 J. A. Pathak and S. D. Hudson, Macromolecules, 2006, 39, 87828792 .

24 J. Soulages and G. H. McKinley, in XVth International Congress on Rheology - the Society of Rheology 80th Annual Meeting, Pts 1 and 2, ed. A. Co, L. G. Leal, R. H. Colby and A. J. Giacomin, Amer Inst Physics, Melville, 2008, vol. 1027, pp. 973-975.

25 J. P. Rothstein, in Rheology Reviews, ed. D. M. Binding and K. Walters, The British Society of Rheology, Aberystwyth, 2008, vol. 6, pp. 1-46.

26 S. Lerouge and J.-F. Berret, Adv. Polym. Sci., 2009, 230, 1-71.

27 R. G. Larson, Rheol. Acta, 1992, 31, 213-263.

28 A. N. Morozov and W. van Saarloos, Phys. Rep., 2007, 447, 112-143.

29 R. Makhloufi, J. P. Decruppe, A. Ait-Ali and R. Cressely, Europhys. Lett., 1995, 32, 253-258.

30 S. J. Muller, Korea-Australia Rheology Journal, 2008, 20, 117-125.

31 Y. Hu, S. Q. Wang and A. M. Jamieson, J. Phys. Chem., 1994, 98, $8555-8559$.

32 R. K. Prud'homme and G. G. Warr, Langmuir, 1994, 10, 3419-3426.

33 L. M. Walker, P. Moldenaers and J.-F. Berret, Langmuir, 1996, 12, 6309-6314.

34 C.-M. Chen and G. G. Warr, Langmuir, 1997, 13, 1374-1376.

35 P. G. De Gennes, J. Chem. Phys., 1974, 60, 5030-5042.

36 D. E. Smith and S. Chu, Science, 1998, 281, 1335-1340.

37 J. A. Odell and A. Keller, J. Polym. Sci., Part B: Polym. Phys., 1986, 24, 1889-1916.

38 J. A. Odell, A. Keller and A. J. Muller, in Polymers in Aqueous Media, ed. J. E. Glass, American Chemical Society, Washington, 1989, vol. 193-244.

39 T. T. Perkins, D. E. Smith and S. Chu, Science, 1997, 276, 2016-2021.

40 P. A. Stone, S. D. Hudson, P. Dalhaimer, D. E. Discher, E. J. Amis and K. B. Migler, Macromolecules, 2006, 39, 7144-7148.

41 O. Scrivener, C. Berner, R. Cressely, R. Hocquart, R. Sellin and N. S. Vlachos, J. Non-Newtonian Fluid Mech., 1979, 5, 475-495.

42 P. E. Arratia, C. C. Thomas, J. Diorio and J. P. Gollub, Phys. Rev. Lett., 2006, 96, 144502.

43 T. M. Squires and S. R. Quake, Rev. Mod. Phys., 2005, 77, 977-1026.

44 R. J. Poole, M. A. Alves and P. J. Oliveira, Phys. Rev. Lett., 2007, 99, 164503.

45 G. N. Rocha, R. J. Poole, M. A. Alves and P. J. Oliveira, J. NonNewtonian Fluid Mech., 2009, 156, 58-69.

46 L. Xi and M. D. Graham, J. Fluid Mech., 2009, 622, 145-165. 
47 A. Groisman, M. Enzelberger and S. R. Quake, Science, 2003, 300, 955-958.

48 A. Groisman and S. R. Quake, Phys. Rev. Lett., 2004, 92, 094501.

49 A. Groisman and V. Steinberg, Nature, 2001, 410, 905-908.

50 S. J. Haward, J. A. Odell, Z. Li and X.-F. Yuan, Rheol. Acta, 2010, 49, 781-788.

51 S. J. Haward, Rheol. Acta, 2010, 49, 1219-1225.

52 S. J. Haward, J. A. Odell, Z. Li and X.-F. Yuan, Rheol. Acta, 2010, 49, 633-645.

53 S. J. Haward, V. Sharma and J. A. Odell, Soft Matter, 2011, 7, 9908, DOI: $10.1039 / \mathrm{C} 1 \mathrm{SM} 05493 \mathrm{G}$.

54 H. Rehage and H. Hoffman, J. Phys. Chem., 1988, 92, 4712-4719.

55 T. J. Ober, J. Soulages and G. H. McKinley, J. Rheol., 2011, 55, 11271159.

56 M. S. Turner and M. E. Cates, Langmuir, 1991, 7, 1590-1594.

57 C. J. Pipe, T. S. Majmudar and G. H. McKinley, Rheol. Acta, 2008, 47, 621-642.

58 R. W. Mair and P. T. Callaghan, Europhys. Lett., 1996, 36, 719-724.

59 J. Y. Lee, G. G. Fuller, N. E. Hudson and X.-F. Yuan, J. Rheol., 2005, 49, 537-550.

60 P. T. Callaghan, M. E. Cates, C. J. Rofe and J. B. A. F. Smeulders, J. Phys. II, 1996, 6, 375-393.

61 R. B. Bird, R. C. Armstrong and O. Hassager, Dynamics of Polymeric Liquids, John Wiley and Sons, New York, 1987.

62 S. L. Anna and G. H. McKinley, J. Rheol., 2001, 45, 115-138.

63 N. J. Kim, C. J. Pipe, K. H. Ahn, S. J. Lee and G. H. McKinley, Korea-Australia Rheology Journal, 2010, 22, 31-41.

64 S. H. Spiegelberg, D. C. Ables and G. H. McKinley, J. NonNewtonian Fluid Mech., 1996, 64, 229-267.

65 V. M. Entov and E. J. Hinch, J. Non-Newtonian Fluid Mech., 1997, 72, $31-53$.

66 B. Akers and A. Belmonte, J. Non-Newtonian Fluid Mech., 2006, 135, 97-108.

67 C. D. Meinhart, S. T. Wereley and M. H. B. Gray, Meas. Sci. Technol., 2000, 11, 809-814.

68 J. A. Odell, in Handbook of Experimental Fluid Mechanics, ed. C. Tropea, Y. L. Yarin and J. F. Foss, Springer-Verlag, Heidelberg, 2007, pp. 724-732.
69 G. G. Fuller, Optical Rheometry of Complex Fluids, Oxford University Press, New York, 1995.

70 Y. T. Hu and A. Lips, J. Rheol., 2005, 49, 1001-1027.

71 M. Shribak and R. Oldenbourg, Appl. Opt., 2003, 42, 3009-3017.

72 T. J. Craven, J. M. Rees and W. B. Zimmerman, Microfluid. Nanofluid., 2010, 9, 559-571.

73 F. M. White, Viscous Fluid Flow, McGraw-Hill, New York, 1991.

74 M. Mackley, Rheol. Acta, 2010, 49, 443-458.

75 P. J. Oliveira, F. T. Pinho and G. A. Pinto, J. Non-Newtonian Fluid Mech., 1998, 79, 1-43.

76 P. J. Oliveira and F. T. Pinho, Numer. Heat Transfer, Part B, 1999, 35, 295-315.

77 M. S. N. Oliveira, M. A. Alves, F. T. Pinho and G. H. McKinley, Exp. Fluids, 2007, 43, 437-451.

78 M. S. N. Oliveira, L. E. Rodd, G. H. McKinley and M. A. Alves, Microfluid. Nanofluid., 2008, 5, 809-826.

79 M. A. Alves, P. J. Oliveira and F. T. Pinho, Int. J. Numer. Methods Fluids, 2003, 41, 47-75.

80 G. W. C. Kaye and T. H. Laby, Tables Of Physical And Chemical Constants And Some Mathematical Functions Longman, New York, 1995.

81 R. W. Mair and P. T. Callaghan, J. Rheol., 1997, 41, 901-924.

82 C. Masselon, J.-B. Salmon and A. Colin, Phys. Rev. Lett., 2008, 100, 038301.

83 P. Nghe, G. Degre, P. Tabeling and A. Ajdari, Appl. Phys. Lett., 2008, 93, 204102.

84 S. Chono and Y. Iemoto, J. Rheol., 1990, 34, 295-308.

85 S. Chono and Y. Iemoto, J. Rheol., 1992, 36, 335-356.

86 S. Gulati, D. Liepmann and S. J. Muller, Phys. Rev. E: Stat., Nonlinear, Soft Matter Phys., 2008, 78, 036314.

87 S. Gulati, C. S. Dutcher, D. Liepmann and S. J. Muller, J. Rheol., 2010, 54, 375-392.

88 M. A. Fardin, D. Lopez, J. Croso, G. Gregoire, O. Cardoso, G. H. McKinley and S. Lerouge, Phys. Rev. Lett., 2010, 104, 178303.

89 A. Bhardwaj, E. Miller and J. P. Rothstein, J. Rheol., 2007, 51, 693719.

90 G. R. Moss and J. P. Rothstein, J. Non-Newtonian Fluid Mech., 2010, 165, 1505-1515. 